\title{
A LEGENDRE SPECTRAL COLLOCATION METHOD FOR THE BIHARMONIC DIRICHLET PROBLEM
}

\author{
BERNARD Bialecki ${ }^{1}$ AND ANDREAS KARAGEORGHis ${ }^{2}$
}

\begin{abstract}
A Legendre spectral collocation method is presented for the solution of the biharmonic Dirichlet problem on a square. The solution and its Laplacian are approximated using the set of basis functions suggested by Shen, which are linear combinations of Legendre polynomials. A Schur complement approach is used to reduce the resulting linear system to one involving the approximation of the Laplacian of the solution on the two vertical sides of the square. The Schur complement system is solved by a preconditioned conjugate gradient method. The total cost of the algorithm is $O\left(N^{3}\right)$. Numerical results demonstrate the spectral convergence of the method.
\end{abstract}

Mathematics Subject Classification. 65N35, 65N22.

Received: March 17, 1999. Revised: November 23, 1999.

\section{INTRODUCTION}

The numerical solution of fourth order problems by spectral methods has been the subject of numerous studies in recent years. A review of various spectral formulations for fourth order problems in one and two dimensions is given in [1]. Spectral collocation methods have been particularly popular in applications to physical problems since, in contrast to spectral Galerkin methods, they do not require the evaluation or approximation of integrals. In [2], spectral collocation methods are studied for the solution of a one dimensional fourth order problem. In [4], a Legendre spectral collocation method is proposed and analyzed for the biharmonic equation on a square. However, no algorithm for the solution of the corresponding approximate problem is discussed. The improvement in the poor conditioning of the spectral discretization of the biharmonic equation is examined in [10]. A Chebyshev spectral collocation method is applied to the driven cavity problem in [16]. The application of Chebyshev spectral collocation methods with domain decomposition to the steady-state Navier-Stokes equations (stream function formulation) in complex geometries is investigated in [11,13]. In [12], a fully conforming Chebyshev spectral collocation scheme is developed for the biharmonic equation in two and three dimensions. Finally, a spectral collocation method has been applied to fourth order problems in circular domains in [14]. Further references to the application of spectral methods to fourth order problems can be found in [7] and [3]. The formulation of the biharmonic Legendre spectral collocation problem in this paper and the method of its solution are similar to those developed in [15] and [5] for orthogonal spline collocation with piecewise Hermite bicubics.

Keywords and phrases. Biharmonic Dirichlet problem, spectral collocation, Schur complement, preconditioned conjugate gradient method.

${ }^{1}$ Department of Mathematical and Computer Sciences, Colorado School of Mines, Golden, Colorado 80401, U.S.A.

e-mail: bbialeck@mines.edu

${ }^{2}$ Department of Mathematics and Statistics, University of Cyprus, P.O. Box 537, 1678 Nicosia, Cyprus. 
In this study we consider the biharmonic Dirichlet problem

$$
\Delta^{2} u=f \text { in } \Omega, \quad u=\partial u / \partial n=0 \text { on } \partial \Omega
$$

where $\Delta$ denotes the Laplacian, $\Omega=(-1,1) \times(-1,1), \partial \Omega$ is the boundary of $\Omega$, and $\partial / \partial n$ is the outer normal derivative on $\partial \Omega$.

In contrast to [4], we use the mixed formulation of (1.1) to obtain approximations to both $u$ and $\Delta u$. Specifically, we set $v=\Delta u$ and discretize a coupled pair of Poisson's equations in $u$ and $v$ using a Legendre spectral collocation method with polynomials of degree $\leq N$. As collocation points we take the nodes of the $N$ - 1-point Legendre-Gauss quadrature rather than the Legendre-Gauss-Lobatto points ( $c f$. [4]). Employing a Schur complement approach, we reduce the collocation problem to a Schur complement system involving an approximation to $v$ on the two vertical sides of $\partial \Omega$ and an auxiliary collocation problem for a related biharmonic problem with $v$, instead of $\partial u / \partial n$, specified on the two vertical sides of $\partial \Omega$. The matrix in the Schur complement system is symmetric and positive definite. (This is not the case when the Legendre-Gauss-Lobatto nodes are used as collocation points). Consequently, the Schur complement system is solved by the preconditioned conjugate gradient $(\mathrm{PCG})$ method. A preconditioner is obtained from the auxiliary collocation problem. We conjecture that the preconditioner is spectrally equivalent to the Schur complement matrix. The cost of multiplying the Schur complement matrix by a vector and the cost of solving a linear system with the preconditioner are $O\left(N^{2}\right)$ each. With the number of PCG iterations proportional to $\log N$, the cost of solving the Schur complement system is therefore $O\left(N^{2} \log N\right)$. The solution of the auxiliary collocation problem is obtained with cost $O\left(N^{3}\right)$ using separation of variables and the solution of a simple generalized eigenvalue problem which reduces to two symmetric eigenvalue problems with tridiagonal matrices. The total cost of our algorithm is therefore $O\left(N^{3}\right)$. The algorithm is well suited for parallel implementation since many of its steps involve independent matrixvector multiplications. Numerical results demonstrate the spectral convergence rate of the approximations to $u$ and $v$ in the maximum norm. In comparison, the method of [5], the cost of which is $O\left(N^{2} \log N\right)$ on an $N \times N$ partition, yields fourth order approximations to $u$ and $v$.

In Section 2 we introduce three polynomial spaces, the corresponding basis functions, and collocation matrices. In Section 3 we develop an efficient method for solving a 1-d spectral collocation problem. The biharmonic spectral collocation problem and its solution are discussed in Sections 4 and 5, respectively. Numerical results and conclusions are given in Sections 6 and 7, respectively.

\section{PRELiminaries}

For $N \geq 4$, let $\left\{\xi_{i}\right\}_{i=1}^{N-1}$ and respectively $\left\{w_{i}\right\}_{i=1}^{N-1}$ be the nodes and weights of the $N$-1-point Legendre-Gauss quadrature on $(-1,1)$, and let

$$
D=\operatorname{diag}\left(w_{1}, \ldots, w_{N-1}\right)
$$

For $p$ and $q$ defined on $\left\{\xi_{i}\right\}_{i=1}^{N-1}$, let

$$
\langle p, q\rangle=\sum_{i=1}^{N-1} w_{i}(p q)\left(\xi_{i}\right) .
$$

It follows from the exactness property of the Legendre-Gauss quadrature that

$$
\langle p, q\rangle=\int_{-1}^{1}(p q)(x) \mathrm{d} x, \quad p q \in P_{2 N-3},
$$


where $P_{k}$ denotes the set of polynomials of degree $\leq k$ on $(-1,1)$. Lemma 3.1 in [8] implies also that

$$
-\left\langle p^{\prime \prime}, q\right\rangle=\int_{-1}^{1}\left(p^{\prime} q^{\prime}\right)(x) \mathrm{d} x-\left.p^{\prime} q\right|_{-1} ^{1}+C_{N} p^{(N)} q^{(N)}, \quad p, q \in P_{N},
$$

where $C_{N}$ denotes a generic positive constant that may depend on $N$.

Let

$$
P_{N}^{0}=\left\{p \in P_{N}: p( \pm 1)=0\right\}, \quad P_{N}^{00}=\left\{p \in P_{N}^{0}: p^{\prime}( \pm 1)=0\right\}
$$

(Note that the dimensions of $P_{N}, P_{N}^{0}$, and $P_{N}^{00}$ are $N+1, N-1$, and $N-3$, respectively.) Following (2.7) and (3.4) of [17], we introduce the basis $\left\{\phi_{k}\right\}_{k=2}^{N}$ for $P_{N}^{0}$ and the basis $\left\{\psi_{k}\right\}_{k=4}^{N}$ for $P_{N}^{00}$ with

$$
\begin{gathered}
\phi_{k}(x)=c_{k}\left[L_{k-2}(x)-L_{k}(x)\right], \quad k=2, \ldots, N, \\
\psi_{k}(x)=d_{k}\left[L_{k-4}(x)+a_{k} L_{k-2}(x)+b_{k} L_{k}(x)\right], \quad k=4, \ldots, N,
\end{gathered}
$$

where $L_{k}(x)$ is the $k$ th degree Legendre polynomial normalized by $\int_{-1}^{1} L_{k}^{2}(x) \mathrm{d} x=2 /(2 k+1)$, and

$$
c_{k}=\frac{1}{\sqrt{4 k-2}}, \quad d_{k}=\frac{1}{\sqrt{2(2 k-5)^{2}(2 k-3)}}, \quad a_{k}=-2 \frac{2 k-3}{2 k-1}, \quad b_{k}=\frac{2 k-5}{2 k-1} .
$$

Augmenting the basis $\left\{\psi_{k}\right\}_{k=4}^{N}$ for $P_{N}^{00}$ by $\psi_{2}, \psi_{3} \in P_{N}^{0}$ such that

$$
\psi_{2}^{\prime}(-1)=1, \quad \psi_{2}^{\prime}(1)=0, \quad \psi_{3}^{\prime}(-1)=0, \quad \psi_{3}^{\prime}(1)=1,
$$

we obtain the basis $\left\{\psi_{k}\right\}_{k=2}^{N}$ for $P_{N}^{0}$. Since $\psi_{2}, \psi_{3} \in P_{N}^{0}$ and since $\left\{\phi_{k}\right\}_{k=2}^{N}$ is a basis for $P_{N}^{0}$,

$$
\psi_{2}(x)=\sum_{k=2}^{N} \alpha_{k} \phi_{k}(x), \quad \psi_{3}(x)=\sum_{k=2}^{N} \beta_{k} \phi_{k}(x)
$$

for some $\left\{\alpha_{k}\right\}_{k=2}^{N}$ and $\left\{\beta_{k}\right\}_{k=2}^{N}$. Later we will consider a particular choice of $\left\{\alpha_{k}\right\}_{k=2}^{N}$ and

$$
\beta_{k}=(-1)^{k-1} \alpha_{k}, \quad k=2, \ldots, N
$$

Using (2.5)-(2.7) it is easy to verify that

$$
\psi_{k}(x)=d_{k}\left[c_{k-2}^{-1} \phi_{k-2}(x)-b_{k} c_{k}^{-1} \phi_{k}(x)\right], \quad k=4, \ldots, N .
$$

Thus it follows from $(2.9)-(2.11)$ that

$$
\left[\psi_{2}(x), \ldots, \psi_{N}(x)\right]=\left[\phi_{2}(x), \ldots, \phi_{N}(x)\right] M,
$$

where the nonsingular matrix $M$ has the structure shown in Figure 1.

Augmenting the basis $\left\{\psi_{k}\right\}_{k=2}^{N}$ for $P_{N}^{0}$ by

$$
\psi_{0}(x)=\frac{1}{2}\left[L_{0}(x)-L_{1}(x)\right], \quad \psi_{1}(x)=\frac{1}{2}\left[L_{0}(x)+L_{1}(x)\right],
$$




$$
\left[\begin{array}{rrrrrrr}
\times & -\times & \times & & & & \\
\times & \times & & \times & & & \\
\times & -\times & \times & & \times & & \\
\vdots & \vdots & & \ddots & & \ddots & \\
\times & -\times & & & \times & & \times \\
\times & \times & & & & \times & \\
\times & -\times & & & & & \times
\end{array}\right]
$$

FiguRE 1. Structure of the $(N-1) \times(N-1)$ matrix $M$.

we obtain the basis $\left\{\psi_{k}\right\}_{k=0}^{N}$ for $P_{N}$, where

$$
\psi_{0}(-1)=1, \quad \psi_{0}(1)=0, \quad \psi_{1}(-1)=0, \quad \psi_{1}(1)=1 .
$$

Let

$$
\begin{array}{cc}
A_{\psi}=\left(-\psi_{k}^{\prime \prime}\left(\xi_{i}\right)\right)_{i=1, k=2}^{N-1, N}, & B_{\psi}=\left(\psi_{k}\left(\xi_{i}\right)\right)_{i=1, k=2}^{N-1, N}, \\
A_{\psi, t}=\left(-\psi_{k}^{\prime \prime}\left(\xi_{i}\right)\right)_{i=1, k=0}^{N-1,1}, & B_{\psi, t}=\left(\psi_{k}\left(\xi_{i}\right)\right)_{i=1, k=0}^{N-1,1}, \\
A_{\phi}=\left(-\phi_{k}^{\prime \prime}\left(\xi_{i}\right)\right)_{i=1, k=2}^{N-1, N}, & B_{\phi}=\left(\phi_{k}\left(\xi_{i}\right)\right)_{i=1, k=2}^{N-1, N},
\end{array}
$$

where $i$ and $k$ are the row and column indices, respectively. Clearly, (2.16) and (2.13) imply

$$
A_{\psi, t}=0 \text {. }
$$

It follows from $(2.15),(2.17)$, and (2.12) that

$$
A_{\psi}=A_{\phi} M, \quad B_{\psi}=B_{\phi} M
$$

Let

$$
A_{\phi}^{\prime}=B_{\phi}^{T} D A_{\phi}, \quad B_{\phi}^{\prime}=B_{\phi}^{T} D B_{\phi},
$$

where $D$ is given by (2.1). Clearly, $B_{\phi}^{\prime}$ is symmetric and positive definite.

Lemma 2.1. The matrix $B_{\phi}^{\prime}$ has the structure shown in Figure 2 and

$$
A_{\phi}^{\prime}=\operatorname{diag}(1, \ldots, 1, \times)
$$

where the element $\times$ is positive.

Proof. Equations $(2.20),(2.17)$, and $(2.1)$, imply that the coefficients of $B_{\phi}^{\prime}=\left(b_{k, l}^{\prime}\right)_{k, l=2}^{N}$ and $A_{\phi}^{\prime}=\left(a_{k, l}^{\prime}\right)_{k, l=2}^{N}$ are given by the formulas

$$
b_{k, l}^{\prime}=\sum_{i=1}^{N-1} w_{i}\left(\phi_{k} \phi_{l}\right)\left(\xi_{i}\right), \quad a_{k, l}^{\prime}=-\sum_{i=1}^{N-1} w_{i}\left(\phi_{k}^{\prime \prime} \phi_{l}\right)\left(\xi_{i}\right), \quad k, l=2, \ldots, N .
$$




$$
\left[\begin{array}{ccccccc}
\times & & \times & & & & \\
& \times & & \times & & & \\
\times & & \times & & \times & & \\
& \ddots & & \ddots & & \ddots & \\
& & \times & & \times & & \times \\
& & & \times & & \times & \\
& & & & \times & & \times
\end{array}\right]
$$

FiguRE 2. Structure of the $(N-1) \times(N-1)$ matrix $B_{\phi}^{\prime}$.

Hence it follows from (2.2), (2.3), and Lemma 2.1 in [17] that in order to prove both claims it suffices to show that

$$
b_{N, N-1}^{\prime}=0, \quad a_{N, N}^{\prime}>0
$$

For $N$ odd $(N-1$ even), we have

$$
b_{N, N-1}=\sum_{i=1}^{(N-1) / 2} w_{i}\left(\phi_{N} \phi_{N-1}\right)\left(\xi_{i}\right)+\sum_{i=1}^{(N-1) / 2} w_{i}\left(\phi_{N} \phi_{N-1}\right)\left(-\xi_{i}\right)=0,
$$

where the first identity follows from the symmetry of $\left\{w_{i}\right\}_{i=1}^{N-1}$ and the antisymmetry of $\left\{\xi_{i}\right\}_{i=1}^{N-1}$ about 0 , and the second identity follows from $\left(\phi_{N} \phi_{N-1}\right)(-x)=-\left(\phi_{N} \phi_{N-1}\right)(x)$ which is a consequence of $(2.5)$ and (2.7).

For $N$ even $(N-1$ odd), using arguments similar to those for odd $N$, we have

$$
b_{N, N-1}=\sum_{i=1}^{(N-2) / 2} w_{i}\left(\phi_{N} \phi_{N-1}\right)\left(\xi_{i}\right)+\sum_{i=1}^{(N-2) / 2} w_{i}\left(\phi_{N} \phi_{N-1}\right)\left(-\xi_{i}\right)+\left(\phi_{N} \phi_{N-1}\right)(0) w_{1+(N-2) / 2}=0
$$

where in the last step we also used $\phi_{N-1}(0)=0$ which follows from $(2.5)$ and $L_{k}(0)=0$ for odd $k$.

Finally, (2.4) applied to $p=q=\phi_{N}$ and (2.2) imply $a_{N, N}^{\prime}>0$.

Let

$$
A_{\psi}^{\prime}=B_{\phi}^{T} D A_{\psi}, \quad B_{\psi}^{\prime}=B_{\phi}^{T} D B_{\psi}
$$

Then it follows from (2.19) and (2.20) that

$$
A_{\psi}^{\prime}=A_{\phi}^{\prime} M, \quad B_{\psi}^{\prime}=B_{\phi}^{\prime} M
$$

Let

$$
B_{\psi, t}^{\prime}=B_{\phi}^{T} D B_{\psi, t}
$$

Then (2.17), (2.1), (2.16), (2.2), (2.3), (2.5), (2.13), and orthogonality of the Legendre polynomials imply that that $B_{\psi, t}^{\prime}$ has the structure shown in Figure 3 . Let

$$
A_{\psi, e}=\left[A_{\psi, t} \mid A_{\psi}\right], \quad B_{\psi, e}=\left[B_{\psi, t} \mid B_{\psi}\right]
$$




$$
\left[\begin{array}{cc}
\times & \times \\
\times & \times \\
0 & 0 \\
\vdots & \vdots \\
0 & 0
\end{array}\right]
$$

FiguRE 3. Structure of the $(N-1) \times 2$ matrix $B_{\psi, t}^{\prime}$.

and let

$$
A_{\psi, e}^{\prime}=B_{\phi}^{T} D A_{\psi, e}, \quad B_{\psi, e}^{\prime}=B_{\phi}^{T} D B_{\psi, e} .
$$

Then it follows from (2.18), (2.24), (2.22), and (2.23) that

$$
A_{\psi, e}^{\prime}=\left[0 \mid A_{\phi}^{\prime} M\right], \quad B_{\psi, e}^{\prime}=\left[B_{\psi, t}^{\prime} \mid B_{\phi}^{\prime} M\right] .
$$

\section{1-D Spectral Collocation Problem}

Solving the biharmonic problem requires the solution of the following 1-d spectral collocation problem.

For given $\lambda>0,\left\{g_{i}\right\}_{i=1}^{N-1},\left\{f_{i}\right\}_{i=1}^{N-1}, \alpha$, and $\beta$, consider the problem of finding $p \in P_{N}^{0}$ and $q \in P_{N}$ such that

$$
\begin{aligned}
& p\left(\xi_{i}\right)-\lambda p^{\prime \prime}\left(\xi_{i}\right)+\lambda q\left(\xi_{i}\right)=g_{i}, \quad i=1, \ldots, N-1, \\
& q\left(\xi_{i}\right)-\lambda q^{\prime \prime}\left(\xi_{i}\right)=f_{i}, \quad i=1, \ldots, N-1 . \\
& p^{\prime}(-1)=\alpha, \quad p^{\prime}(1)=\beta \text {. }
\end{aligned}
$$

Theorem 3.1. For $\lambda>0$, there exist unique $p \in P_{N}^{0}$ and $q \in P_{N}$ satisfying (3.1)-(3.3).

Proof. Since in (3.1)-(3.3), the number of unknowns, which is $2 N$, is equal to the number of equations, we assume $g_{i}=f_{i}=0, i=1, \ldots, 2 N, \alpha=\beta=0$ and show that $p=q=0$. Taking the inner product $\langle\cdot, \cdot\rangle$ with $q$ on both sides of (3.1) and with $p$ on both sides of (3.2), respectively, we obtain

$$
\begin{aligned}
\langle p, q\rangle-\lambda\left\langle p^{\prime \prime}, q\right\rangle+\lambda\langle q, q\rangle & =0, \\
\langle q, p\rangle-\lambda\left\langle q^{\prime \prime}, p\right\rangle & =0 .
\end{aligned}
$$

It follows from (2.4) and $p( \pm 1)=p^{\prime}( \pm 1)=0$ that $\left\langle p^{\prime \prime}, q\right\rangle=\left\langle q^{\prime \prime}, p\right\rangle$. Hence (3.4) gives $\langle q, q\rangle=0$, which implies

$$
q\left(\xi_{i}\right)=0, \quad i=1, \ldots, N-1 .
$$

From (3.2) and (3.5), we have $q^{\prime \prime}\left(\xi_{i}\right)=0, i=1, \ldots, N-1$, which yields $q^{\prime \prime}=0$ since $q^{\prime \prime} \in P_{N-2}$. Thus $q \in P_{1}$ and hence (3.5) and $N \geq 3$ imply $q=0$.

From (3.1) and (3.5), we have

$$
\langle p, p\rangle-\lambda\left\langle p^{\prime \prime}, p\right\rangle=0 .
$$

Since, by $(2.4),-\left\langle p^{\prime \prime}, p\right\rangle \geq 0$, it follows that $\langle p, p\rangle=0$. Thus $p\left(\xi_{i}\right)=0, i=1, \ldots, N-1$, which along with $p( \pm 1)=0$ implies $p=0$. 
In the remainder of this section we consider a matrix-vector form of (3.1)-(3.3), assuming that $\left\{\psi_{k}\right\}_{k=2}^{N}$ and $\left\{\psi_{k}\right\}_{k=0}^{N}$ are respectively bases for $P_{N}^{0}$ and $P_{N}$ introduced in Section 2. Substituting

$$
p(x)=\sum_{k=2}^{N} p_{k} \psi_{k}(x), \quad q(x)=\sum_{k=0}^{N} q_{k} \psi_{k}(x),
$$

into (3.1)-(3.3), and using (2.15), (2.16), (2.25), (2.8), and $\psi_{k}^{\prime}( \pm 1)=0, k=4, \ldots, N$, we obtain

$$
\left(B_{\psi}+\lambda A_{\psi}\right) \vec{p}+\lambda B_{\psi, e} \vec{q}_{e}=\vec{g}, \quad\left(B_{\psi, e}+\lambda A_{\psi, e}\right) \vec{q}_{e}=\vec{f}, \quad p_{2}=\alpha, \quad p_{3}=\beta,
$$

where

$$
\begin{array}{cc}
\vec{p}=\left[p_{2}, \ldots, p_{N}\right]^{T}, & \vec{q}_{e}=\left[q_{0}, \ldots, q_{N}\right]^{T}, \\
\vec{g}=\left[g_{1}, \ldots, g_{N-1}\right]^{T}, \quad \vec{f}=\left[f_{1}, \ldots, f_{N-1}\right]^{T} .
\end{array}
$$

Multiplying the first two equations of (3.6) by $B_{\phi}^{T} D$, with $B_{\phi}$ and $D$ defined in (2.17) and (2.1), respectively, and using (2.22), (2.26), we obtain

$$
\left(B_{\psi}^{\prime}+\lambda A_{\psi}^{\prime}\right) \vec{p}+\lambda B_{\psi, e}^{\prime} \vec{q}_{e}=\vec{g}_{\phi}, \quad\left(B_{\psi, e}^{\prime}+\lambda A_{\psi, e}^{\prime}\right) \vec{q}_{e}=\vec{f}_{\phi}, \quad p_{2}=\alpha, \quad p_{3}=\beta,
$$

where

$$
\vec{g}_{\phi}=B_{\phi}^{T} D \vec{g}, \quad \vec{f}_{\phi}=B_{\phi}^{T} D \vec{f}
$$

Rewriting $\vec{q}_{e}$ of (3.7) as

$$
\vec{q}_{e}=\left[q_{0}, q_{1}, \vec{q}\right]^{T}, \quad \vec{q}=\left[q_{2}, \ldots, q_{N}\right]^{T},
$$

and using (2.23), (2.27), (2.8), $\psi_{k}^{\prime}( \pm 1)=0, k=4, \ldots, N$, and (2.12), we obtain

$$
\begin{aligned}
\left(B_{\phi}^{\prime}+\lambda A_{\phi}^{\prime}\right) \vec{p}_{M}+\lambda B_{\phi}^{\prime} \vec{q}_{M}+\lambda B_{\psi, t}^{\prime}\left[q_{0}, q_{1}\right]^{T} & =\vec{g}_{\phi}, \\
\left(B_{\phi}^{\prime}+\lambda A_{\phi}^{\prime}\right) \vec{q}_{M}+B_{\psi, t}^{\prime}\left[q_{0}, q_{1}\right]^{T} & =\vec{f}_{\phi}, \\
C_{\phi} \vec{p}_{M} & =[\alpha, \beta]^{T},
\end{aligned}
$$

where

$$
\vec{p}_{M}=M \vec{p}, \quad \vec{q}_{M}=M \vec{q},
$$

and

$$
C_{\phi}=\left[\begin{array}{llll}
\phi_{2}^{\prime}(-1) & \phi_{3}^{\prime}(-1) & \ldots & \phi_{N}^{\prime}(-1) \\
\phi_{2}^{\prime}(1) & \phi_{3}^{\prime}(1) & \ldots & \phi_{N}^{\prime}(1)
\end{array}\right]
$$

Equations (3.10) can be rewritten as

$$
\begin{aligned}
S_{11}\left[\vec{p}_{M}, \vec{q}_{M}\right]^{T}+S_{12}\left[q_{0}, q_{1}\right]^{T} & =\left[\vec{g}_{\phi}, \vec{f}_{\phi}\right]^{T}, \\
S_{21}\left[\vec{p}_{M}, \vec{q}_{M}\right]^{T} & =[\alpha, \beta]^{T},
\end{aligned}
$$


where

$$
S_{11}=\left[\begin{array}{cc}
B_{\phi}^{\prime}+\lambda A_{\phi}^{\prime} & \lambda B_{\phi}^{\prime} \\
O & B_{\phi}^{\prime}+\lambda A_{\phi}^{\prime}
\end{array}\right]
$$

and

$$
S_{12}=\left[\begin{array}{c}
\lambda B_{\psi, t}^{\prime} \\
B_{\psi, t}^{\prime}
\end{array}\right], \quad S_{21}=\left[\begin{array}{cc}
C_{\phi} & O
\end{array}\right]
$$

Since $B_{\phi}^{\prime}+\lambda A_{\phi}^{\prime}$ is positive definite, $S_{11}$ is nonsingular. Hence, using (3.12), we obtain

$$
\left[\vec{p}_{M}, \vec{q}_{M}\right]^{T}=S_{11}^{-1}\left[\vec{g}_{\phi}, \vec{f}_{\phi}\right]^{T}-S_{11}^{-1} S_{12}\left[q_{0}, q_{1}\right]^{T},
$$

which upon substitution into (3.13) gives

$$
S\left[q_{0}, q_{1}\right]^{T}=S_{21} S_{11}^{-1}\left[\vec{g}_{\phi}, \vec{f}_{\phi}\right]^{T}-[\alpha, \beta]^{T},
$$

where $S$ is the $2 \times 2$ Schur complement matrix given by

$$
S=S_{21} S_{11}^{-1} S_{12}
$$

Thus we have the following algorithm for solving (3.8), assuming that $\vec{g}_{\phi}$ and $\vec{f}_{\phi}$ are known. (We focus on (3.8), rather than (3.6), since it is (3.8) which arises in the solution of the 2 -d problem.)

\section{Algorithm I.}

Step 1: Compute columns of $S$ using (3.17).

Step 2: Compute the right hand side of (3.16).

Step 3: Solve (3.16) for $\left[q_{0}, q_{1}\right]^{T}$.

Step 4: Compute $\left[\vec{p}_{M}, \vec{q}_{M}\right]^{T}$ using (3.15).

Step 5: Compute $\vec{p}$ and $\vec{q}$ using (3.11).

Note that in steps 1 and 2 we can save $S_{11}^{-1} S_{12}$ and $S_{11}^{-1}\left[\vec{g}_{\phi}, \vec{f}_{\phi}\right]^{T}$ which are used in step 4. By (3.14), solving, in steps 1 and 2, linear systems with $S_{11}$ involves solving two linear systems with $B_{\phi}^{\prime}+\lambda A_{\phi}^{\prime}$ and multiplication by $\lambda B_{\phi}^{\prime}$. It follows from (2.21) and the structure of $B_{\phi}^{\prime}$ (see Fig. 2) that solving a linear system with $B_{\phi}^{\prime}+\lambda A_{\phi}^{\prime}$ reduces to solving two linear systems with tridiagonal symmetric and positive definite matrices. Step 3 involves solving a linear system of two equations in two unknowns. It follows from the structure of $M$ (see Fig. 1) that in step 5, $M \vec{p}=\vec{p}_{M}$ ( similarly $M \vec{q}=\vec{q}_{M}$ ) can be decoupled into two systems, one for $p_{2}-p_{3}$ and $p_{k}$ with even $k \geq 4$, and the other for $p_{2}+p_{3}$ and $p_{k}$ with odd $k \geq 5$. The matrices in these two systems have the structure shown in Figure 4 and hence each system can be solved with cost $O(N)$. Since each step of Algorithm I requires at most $O(N)$ operations, the total cost of solving (3.8) is $O(N)$. Of course the cost of computing $\vec{g}_{\phi}$ and $\vec{f}_{\phi}$ of (3.9) is $O\left(N^{2}\right)$.

\section{Biharmonic SPECTRAL COLlocation PROBlem}

Introducing $v=\Delta u$ in (1.1), we obtain the coupled problem

$$
-\Delta u+v=0 \text { in } \Omega, \quad-\Delta v=-f \text { in } \Omega, \quad u=\partial u / \partial n=0 \text { on } \partial \Omega .
$$




$$
\left[\begin{array}{cccccc}
\times & \times & & & & \\
\times & \times & \times & & & \\
\times & & \times & \times & & \\
\vdots & & & \ddots & \ddots & \\
\times & & & & \times & \times \\
\times & & & & & \times
\end{array}\right]
$$

Figure 4. Structure of the matrices in decoupled systems for $M \vec{p}=\vec{p}_{M}$.

The Legendre spectral collocation problem corresponding to (4.1) consists of finding $U \in P_{N}^{00} \otimes P_{N}^{00}$ and $V \in P_{N} \otimes P_{N}$ such that

$$
-\Delta U\left(\xi_{i}, \xi_{j}\right)+V\left(\xi_{i}, \xi_{j}\right)=0, \quad-\Delta V\left(\xi_{i}, \xi_{j}\right)=-f\left(\xi_{i}, \xi_{j}\right), \quad i, j=1, \ldots, N-1
$$

and

$$
V(a, b)=V_{y}(a, b)=0, \quad a, b= \pm 1
$$

We prove existence and uniqueness of the solution to (4.2)-(4.3) following the proof of Theorem 5.1 in [15]. To this end, we require an additional notation and two lemmas. For $p$ and $q$ defined on $\left\{(x, y): x, y \in\left\{\xi_{i}\right\}_{i=1}^{N-1}\right\}$, let

$$
\langle\langle p, q\rangle\rangle=\sum_{i=1}^{N-1} \sum_{j=1}^{N-1} w_{i} w_{j}(p q)\left(\xi_{i}, \xi_{j}\right)
$$

Lemma 4.1. If $U \in P_{N}^{00} \otimes P_{N}^{00}$ and $V \in P_{N} \otimes P_{N}$, then

$$
\langle\langle-\Delta U, V\rangle\rangle=\langle\langle U,-\Delta V\rangle\rangle
$$

Proof. Since $U_{x}(a, y)=0, a= \pm 1, y \in[-1,1]$, using $(2.4)$, we have

$$
\begin{aligned}
\left\langle\left\langle-U_{x x}, V\right\rangle\right\rangle & =-\sum_{j=1}^{N-1} w_{j}\left\langle U_{x x}\left(\cdot, \xi_{j}\right), V\left(\cdot, \xi_{j}\right)\right\rangle \\
& =\sum_{j=1}^{N-1} w_{j}\left[\int_{-1}^{1}\left(U_{x} V_{x}\right)\left(x, \xi_{j}\right) \mathrm{d} x+C_{N}\left(\frac{\partial^{N} U}{\partial x^{N}} \frac{\partial^{N} V}{\partial x^{N}}\right)\left(\cdot, \xi_{j}\right)\right] .
\end{aligned}
$$

In a similar way, using $(2.4)$ and $U(a, y)=0, a= \pm 1, y \in[-1,1]$, we obtain

$$
\left\langle\left\langle-V_{x x}, U\right\rangle\right\rangle=\sum_{j=1}^{N-1} w_{j}\left[\int_{-1}^{1}\left(V_{x} U_{x}\right)\left(x, \xi_{j}\right) \mathrm{d} x+C_{N}\left(\frac{\partial^{N} V}{\partial x^{N}} \frac{\partial^{N} U}{\partial x^{N}}\right)\left(\cdot, \xi_{j}\right)\right] .
$$

Therefore $\left\langle\left\langle-U_{x x}, V\right\rangle\right\rangle=\left\langle\left\langle U,-V_{x x}\right\rangle\right\rangle$. By symmetry in $x$ and $y$ we also have $\left\langle\left\langle-U_{y y}, V\right\rangle\right\rangle=\left\langle\left\langle U,-V_{y y}\right\rangle\right\rangle$. Hence the desired result follows. 
Lemma 4.2. If $V \in P_{N} \otimes P_{N}$ satisfies

$$
V(a, b)=V_{y}(a, b)=0, \quad a, b= \pm 1,
$$

then

$$
\left\langle\left\langle V_{x x}, V_{y y}\right\rangle\right\rangle=\left\langle\left\langle V, V_{x x y y}\right\rangle\right\rangle .
$$

Proof. Applying (2.4) with respect to $y$ to the left-hand side of (4.6), we have

$$
\begin{aligned}
\left\langle\left\langle V_{x x}, V_{y y}\right\rangle\right\rangle= & \sum_{i=1}^{N-1} w_{i}\left\langle V_{y y}\left(\xi_{i}, \cdot\right), V_{x x}\left(\xi_{n}, \cdot\right)\right\rangle \\
= & -\sum_{i=1}^{N-1} w_{i} \int_{-1}^{1}\left(V_{y} V_{x x y}\right)\left(\xi_{i}, y\right) \mathrm{d} y \\
& +\left.\sum_{i=1}^{N-1} w_{i}\left(V_{y} V_{x x}\right)\left(\xi_{i}, y\right)\right|_{y=-1} ^{y=1}-C_{N} \sum_{i=1}^{N-1} w_{i}\left(\frac{\partial^{N} V}{\partial y^{N}} \frac{\partial^{N+2} V}{\partial y^{N} \partial x^{2}}\right)\left(\xi_{i}, \cdot\right) .
\end{aligned}
$$

Applying (2.4) to the second term on the right-hand side in (4.7), we obtain, for $y= \pm 1$,

$$
\begin{aligned}
\sum_{i=1}^{N-1} w_{i}\left(V_{y} V_{x x}\right)\left(\xi_{i}, y\right) & =\left\langle V_{x x}(\cdot, y), V_{y}(\cdot, y)\right\rangle \\
& =-\int_{-1}^{1}\left(V_{x} V_{y x}\right)(x, y) \mathrm{d} x+\left.\left(V_{x} V_{y}\right)(x, y)\right|_{x=-1} ^{x=1}-C_{N}\left(\frac{\partial^{N} V}{\partial x^{N}} \frac{\partial^{N+1} V}{\partial x^{N} \partial y}\right)(\cdot, y)
\end{aligned}
$$

Applying (2.4) to the first term on the right-hand side in (4.8), we obtain, for $y= \pm 1$,

$$
-\int_{-1}^{1}\left(V_{y x} V_{x}\right)(x, y) \mathrm{d} x=\sum_{i=1}^{N-1} w_{i}\left(V_{y x x} V\right)\left(\xi_{i}, y\right)-\left.\left(V_{y x} V\right)(x, y)\right|_{x=-1} ^{x=1}+C_{N} \sum_{i=1}^{N-1} w_{i}\left(\frac{\partial^{N+1} V}{\partial x^{N} \partial y} \frac{\partial^{N} V}{\partial x^{N}}\right)(\cdot, y) .
$$

Substituting (4.9) into (4.8) and using (4.5), we have

$$
\sum_{i=1}^{N-1} w_{i}\left(V_{y} V_{x x}\right)\left(\xi_{i}, y\right)=\sum_{i=1}^{N-1} w_{i}\left(V_{y x x} V\right)\left(\xi_{i}, y\right), \quad y= \pm 1 .
$$

Applying (2.4) with respect to $y$ to the right-hand side of (4.6), we also obtain

$$
\begin{aligned}
\left\langle\left\langle V, V_{x x y y}\right\rangle\right\rangle= & \sum_{i=1}^{N-1} w_{i}\left\langle V_{x x y y}\left(\xi_{i}, \cdot\right), V\left(\xi_{i}, \cdot\right)\right\rangle \\
= & -\sum_{i=1}^{N-1} w_{i} \int_{-1}^{1}\left(V_{x x y} V_{y}\right)\left(\xi_{i}, y\right) \mathrm{d} y \\
& +\left.\sum_{i=1}^{N-1} w_{i}\left(V_{x x y} V\right)\left(\xi_{i}, y\right)\right|_{y=-1} ^{y=1}-C_{N} \sum_{i=1}^{N-1} w_{i} \frac{\partial^{N+2} V}{\partial y^{N} \partial x^{2}} \frac{\partial^{N} V}{\partial y^{N}}\left(\xi_{i}, .\right) .
\end{aligned}
$$

Comparing the right-hand sides of (4.7) and (4.11), and using (4.10), we obtain (4.6).

Theorem 4.3. There exist unique $U \in P_{N}^{00} \otimes P_{N}^{00}$ and $V \in P_{N} \otimes P_{N}$ satisfying (4.2)-(4.3). 
Proof. Since the number of unknown coefficients in $U$ and $V$, which is $N^{2}-4 N+10$, is equal to the number of equations in (4.2)-(4.3), it suffices to show that if $U$ and $V$ satisfy (4.2)-(4.3) with $f=0$, then $U=V=0$.

Taking the inner product $\langle\langle\cdot, \cdot\rangle\rangle$ with $V$ on both sides of the first equation in $(4.2)$, we obtain

$$
\langle\langle-\Delta U, V\rangle\rangle+\langle\langle V, V\rangle\rangle=0 .
$$

Similarly, taking the inner product $\langle\langle\cdot, \cdot\rangle\rangle$ with $U$ on both sides of the second equation in (4.2), we obtain

$$
\langle\langle-\Delta V, U\rangle\rangle=0
$$

From (4.12), (4.13), and Lemma 4.1, we have $\langle\langle V, V\rangle\rangle=0$, which implies

$$
V\left(\xi_{i}, \xi_{j}\right)=0, \quad i, j=1, \ldots, N-1 .
$$

Thus, by the first equation in (4.2),

$$
-\Delta U\left(\xi_{i}, \xi_{j}\right)=0, \quad i, j=1, \ldots, N-1 .
$$

Using this equation, (2.4) with respect to $x$ and $y$, and $U=0$ on $\partial \Omega$, we have

$$
\begin{aligned}
0=\langle\langle-\Delta U, U\rangle\rangle & =-\sum_{j=1}^{N-1} w_{j}\left\langle U_{x x}\left(\cdot, \xi_{j}\right), U\left(\cdot, \xi_{j}\right)\right\rangle-\sum_{i=1}^{N-1} w_{i}\left\langle U_{y y}\left(\xi_{i}, \cdot\right), U\left(\xi_{i}, \cdot\right)\right\rangle \\
& \geq \sum_{j=1}^{N-1} w_{j} \int_{-1}^{1} U_{x}^{2}\left(x, \xi_{j}\right) \mathrm{d} x+\sum_{i=1}^{N-1} w_{i} \int_{-1}^{1} U_{y}^{2}\left(\xi_{i}, y\right) \mathrm{d} y
\end{aligned}
$$

which along with $U=0$ on $\partial \Omega$ implies that $U=0$ on the horizontal and vertical lines passing through the points $\left(\xi_{i}, \xi_{j}\right), i, j=1, \ldots, N-1$. This and $U=0$ on $\partial \Omega$ imply further that $U=0$ on all horizontal and vertical lines passing through $\Omega$, and hence $U=0$.

To show that $V=0$, we use the second equation in (4.2) (with $f=0$ ), Lemma 4.2 , and (4.14) to obtain

$$
\begin{aligned}
0 & =\langle\langle\Delta V, \Delta V\rangle\rangle=\left\langle\left\langle V_{x x}, V_{x x}\right\rangle\right\rangle+2\left\langle\left\langle V_{x x}, V_{y y}\right\rangle\right\rangle+\left\langle\left\langle V_{y y}, V_{y y}\right\rangle\right\rangle \\
& =\left\langle\left\langle V_{x x}, V_{x x}\right\rangle\right\rangle+2\left\langle\left\langle V, V_{x x y y}\right\rangle\right\rangle+\left\langle\left\langle V_{y y}, V_{y y}\right\rangle\right\rangle=\left\langle\left\langle V_{x x}, V_{x x}\right\rangle\right\rangle+\left\langle\left\langle V_{y y}, V_{y y}\right\rangle\right\rangle .
\end{aligned}
$$

Hence

$$
V_{x x}\left(\xi_{i}, \xi_{j}\right)=V_{y y}\left(\xi_{i}, \xi_{j}\right)=0, \quad i, j=1, \ldots, N-1
$$

which along with (4.14) implies that $V=0$ on the horizontal and vertical lines passing through the points $\left(\xi_{i}, \xi_{j}\right), i, j=1, \ldots, N-1$. This and $V(a, b)=0, a, b= \pm 1$, imply in turn that $V=0$ on $\partial \Omega$. Therefore, $V=0$ on all horizontal and vertical lines passing through $\Omega$, and hence $V=0$.

Let the functions $\left\{\psi_{k}\right\}_{k=0}^{N}$ be as in Section 2 with $\left\{\alpha_{k}\right\}_{k=2}^{N}$ of $(2.9)$ yet to be specified and $\left\{\beta_{k}\right\}_{k=2}^{N}$ as in (2.10). Since $\left\{\psi_{k}\right\}_{k=4}^{N},\left\{\psi_{k}\right\}_{k=2}^{N}$, and $\left\{\psi_{k}\right\}_{k=0}^{N}$ form bases for $P_{N}^{00}, P_{N}^{0}$, and $P_{N}$, respectively, and since $\psi_{0}, \psi_{1}$ and $\psi_{2}, \psi_{3}$ satisfy (2.14) and (2.8), respectively, for $U \in P_{N}^{00} \otimes P_{N}^{00}$ and $V \in P_{N} \otimes P_{N}$ satisfying (4.3), we have

$$
U(x, y)=\sum_{k=2}^{N} \sum_{l=4}^{N} u_{k, l} \psi_{k}(x) \psi_{l}(y)
$$

with

$$
u_{2, l}=u_{3, l}=0, \quad l=4, \ldots, N
$$


and

$$
V(x, y)=\sum_{k=2}^{N} \sum_{l=0}^{N} v_{k, l} \psi_{k}(x) \psi_{l}(y)+\sum_{k=0}^{1} \sum_{l=4}^{N} v_{k, l} \psi_{k}(x) \psi_{l}(y) .
$$

Note that in (4.15) we included

$$
\sum_{k=2}^{3} \sum_{l=4}^{N} u_{k, l} \psi_{k}(x) \psi_{l}(y)
$$

assuming (4.16) and we did not include

$$
\sum_{k=0}^{1} \sum_{l=0}^{3} v_{k, l} \psi_{k}(x) \psi_{l}(y)
$$

in (4.17) since (4.3), $\psi_{2}( \pm 1)=\psi_{3}( \pm 1)=0,(2.14)$, and (2.8) imply that all the coefficients in (4.18) are zero.

Corresponding to (4.15) and (4.17) we introduce the vectors

$$
\begin{gathered}
\vec{u}=\left[u_{2,4}, \ldots, u_{2, N}, \ldots, u_{N, 4}, \ldots, u_{N, N}\right]^{T}, \\
\vec{u}_{2, \cdot}=\left[u_{2,4}, \ldots, u_{2, N}\right]^{T}, \quad \vec{u}_{3, \cdot}=\left[u_{3,4}, \ldots, u_{3, N}\right]^{T}, \\
\vec{v}=\left[v_{2,0}, \ldots, v_{2, N}, \ldots, v_{N, 0}, \ldots, v_{N, N}\right]^{T}, \\
\vec{v}_{0, \cdot}=\left[v_{0,4}, \ldots, v_{0, N}\right]^{T}, \quad \vec{v}_{1, .}=\left[v_{1,4}, \ldots, v_{1, N}\right]^{T} .
\end{gathered}
$$

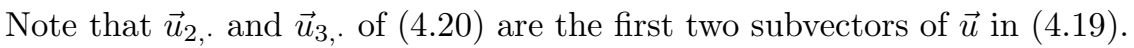

Let $A_{\psi}, B_{\psi}, A_{\psi, t}, B_{\psi, t}$, and $A_{\psi, e}, B_{\psi, e}$ be the matrices introduced in (2.15), (2.16), and (2.25), respectively, and let the two additional matrices $A_{\psi, r}, B_{\psi, r}$ be defined by

$$
A_{\psi, r}=\left(-\psi_{k}^{\prime \prime}\left(\xi_{i}\right)\right)_{i=1, k=4}^{N-1, N}, \quad B_{\psi, r}=\left(\psi_{k}\left(\xi_{i}\right)\right)_{i=1, k=4}^{N-1, N} .
$$

Substituting (4.15) and (4.17) into (4.2), using the matrix definitions, (2.18), and (4.16), we obtain

$$
\begin{gathered}
\left(A_{\psi} \otimes B_{\psi, r}+B_{\psi} \otimes A_{\psi, r}\right) \vec{u}+\left(B_{\psi} \otimes B_{\psi, e}\right) \vec{v}+\left(B_{\psi, t} \otimes B_{\psi, r}\right)\left[\vec{v}_{0, \cdot}, \vec{v}_{1, \cdot}\right]^{T}=\overrightarrow{0}, \\
\left(A_{\psi} \otimes B_{\psi, e}+B_{\psi} \otimes A_{\psi, e}\right) \vec{v}+\left(B_{\psi, t} \otimes A_{\psi, r}\right)\left[\vec{v}_{0, \cdot}, \vec{v}_{1, \cdot}\right]^{T}=\vec{f} \\
\vec{u}_{2, .}=-\vec{u}_{3, .}=\overrightarrow{0},
\end{gathered}
$$

where

$$
\vec{f}=\left[f_{1,1}, \ldots, f_{1, N-1}, \ldots, f_{N-1,1}, \ldots, f_{N-1, N-1}\right]^{T}
$$

with $f_{i, j}=-f\left(\xi_{i}, \xi_{j}\right)$. 


\section{Solving the Biharmonic SPECTRAL COLlOCATion PROBlem}

In this section we present a method for solving (4.24)-(4.26).

\subsection{Formulation of the method}

Let $I_{k}$ be the $k \times k$ identity matrix. Multiplying (4.24) and (4.25) by $B_{\phi}^{T} D \otimes B_{\phi}^{T} D,(4.26)$ by $B_{\psi, r}^{T} D B_{\psi, r}$ and using (2.22)-(2.24), (2.26), we obtain

$$
\begin{gathered}
\left(A_{\phi}^{\prime} \otimes B_{\psi, r}^{\prime}+B_{\phi}^{\prime} \otimes A_{\psi, r}^{\prime}\right)\left(M \otimes I_{N-3}\right) \vec{u}+\left(B_{\phi}^{\prime} \otimes B_{\psi, e}^{\prime}\right)\left(M \otimes I_{N+1}\right) \vec{v}+\left(B_{\psi, t}^{\prime} \otimes B_{\psi, r}^{\prime}\right)\left[\vec{v}_{0, \cdot}, \vec{v}_{1, \cdot}\right]^{T}=\overrightarrow{0} \\
\left(A_{\phi}^{\prime} \otimes B_{\psi, e}^{\prime}+B_{\phi}^{\prime} \otimes A_{\psi, e}^{\prime}\right)\left(M \otimes I_{N+1}\right) \vec{v}+\left(B_{\psi, t}^{\prime} \otimes A_{\psi, r}^{\prime}\right)\left[\vec{v}_{0, \cdot}, \vec{v}_{1, \cdot}\right]^{T}=\vec{f}_{\phi} \\
B_{\psi, r}^{T} D B_{\psi, r} \vec{u}_{2, \cdot}=-B_{\psi, r}^{T} D B_{\psi, r} \vec{u}_{3, \cdot}=\overrightarrow{0}
\end{gathered}
$$

where

$$
\begin{gathered}
A_{\psi, r}^{\prime}=B_{\phi}^{T} D A_{\psi, r}, \quad B_{\psi, r}^{\prime}=B_{\phi}^{T} D B_{\psi, r}, \\
\vec{f}_{\phi}=\left(B_{\phi}^{T} D \otimes B_{\phi}^{T} D\right) \vec{f} .
\end{gathered}
$$

Lemma 5.1. The matrix of the linear system (5.1)-(5.3) is nonsingular.

Proof. Clearly, $B_{\psi}$ of (2.15) is nonsingular since $p=0$ is the only $p \in P_{N}^{0}$ such that $p\left(\xi_{i}\right)=0, i=1, \ldots, N-1$. Hence the rank of the $(N-1) \times(N-3)$ matrix $B_{\psi, r}$ of (4.23) is $N-3$. This implies nonsingularity of the $(N-3) \times(N-3)$ matrix $B_{\psi, r}^{T} D B_{\psi, r}$ since $B_{\psi, r}^{T} D B_{\psi, r} \vec{w}=\overrightarrow{0}$ yields $\vec{w}^{T}\left(D^{1 / 2} B_{\psi, r}\right)^{T} D^{1 / 2} B_{\psi, r} \vec{w}=0, D^{1 / 2} B_{\psi, r} \vec{w}=$ $\overrightarrow{0}, B_{\psi, r} \vec{w}=\overrightarrow{0}$, and $\vec{w}=\overrightarrow{0}$. The desired result follows now from the nonsingularity of $B_{\phi}^{T} D, B_{\psi, r}^{T} D B_{\psi, r}$, and the matrix in (4.24)-(4.26), which is guaranteed by the uniqueness of the solution to (4.2)-(4.3).

Equations (5.1)-(5.3) can be written as

$$
\begin{gathered}
S_{11}[\vec{u}, \vec{v}]^{T}+S_{12}\left[\vec{v}_{0, \cdot}, \vec{v}_{1, \cdot}\right]^{T}=\left[\overrightarrow{0}, \vec{f}_{\phi}\right]^{T}, \\
S_{21}[\vec{u}, \vec{v}]^{T}=\overrightarrow{0}
\end{gathered}
$$

where

$$
\begin{aligned}
& S_{11}=\left[\begin{array}{cc}
\left(A_{\phi}^{\prime} \otimes B_{\psi, r}^{\prime}+B_{\phi}^{\prime} \otimes A_{\psi, r}^{\prime}\right)\left(M \otimes I_{N-3}\right) & \left(B_{\phi}^{\prime} \otimes B_{\psi, e}^{\prime}\right)\left(M \otimes I_{N+1}\right) \\
O & \left(A_{\phi}^{\prime} \otimes B_{\psi, e}^{\prime}+B_{\phi}^{\prime} \otimes A_{\psi, e}^{\prime}\right)\left(M \otimes I_{N+1}\right)
\end{array}\right], \\
& S_{12}=\left[\begin{array}{c}
B_{\psi, t}^{\prime} \otimes B_{\psi, r}^{\prime} \\
B_{\psi, t}^{\prime} \otimes A_{\psi, r}^{\prime}
\end{array}\right] \\
& S_{21}=\left[\begin{array}{ccc}
B_{\psi, r}^{T} D B_{\psi, r} & O & O \\
O & -B_{\psi, r}^{T} D B_{\psi, r} & O
\end{array}\right]
\end{aligned}
$$

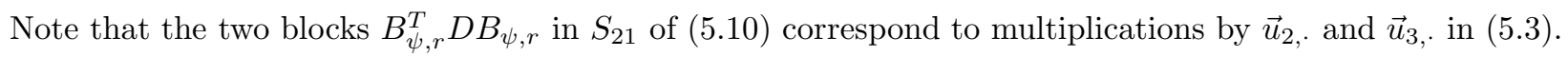


Lemma 5.2. The matrix $S_{11}$ of (5.8) is nonsingular.

Proof. With $\vec{u}$ and $\vec{v}$ of the forms (4.19) and (4.21), respectively, the equations

$$
\left(A_{\psi} \otimes B_{\psi, r}+B_{\psi} \otimes A_{\psi, r}\right) \vec{u}+\left(B_{\psi} \otimes B_{\psi, e}\right) \vec{v}=\overrightarrow{0}, \quad\left(A_{\psi} \otimes B_{\psi, e}+B_{\psi} \otimes A_{\psi, e}\right) \vec{v}=\overrightarrow{0}
$$

are the matrix-vector representations of the following spectral collocation problem: find $U \in P_{N}^{0} \otimes P_{N}^{00}, V \in$ $P_{N}^{0} \otimes P_{N}$, such that

$$
-\Delta U\left(\xi_{i}, \xi_{j}\right)+V\left(\xi_{i}, \xi_{j}\right)=0, \quad-\Delta V\left(\xi_{i}, \xi_{j}\right)=0, \quad i, j=1, \ldots, N-1
$$

It can be shown, using an approach similar to the proof of Theorem 4.3, that the only solution to this problem is $U=V=0$ which gives $\vec{u}=\vec{v}=\overrightarrow{0}$. This and the nonsingularity of $B_{\phi}^{T} D$ imply the nonsingularity of $S_{11}$.

Since $S_{11}$ is nonsingular, eliminating $[\vec{u}, \vec{v}]^{T}$ from $(5.6)-(5.7)$, we obtain

$$
S\left[\vec{v}_{0, \cdot}, \vec{v}_{1, \cdot}\right]^{T}=S_{21} S_{11}^{-1}\left[\overrightarrow{0}, \vec{f}_{\phi}\right]^{T}
$$

where $S$ is the $2(N-3) \times 2(N-3)$ Schur complement matrix given by

$$
S=S_{21} S_{11}^{-1} S_{12}
$$

Lemma 5.3. The matrix $S$ of (5.12) is nonsingular.

Proof. The matrix $S$ is nonsingular since it is the Schur complement of the nonsingular $S_{11}$ (see Lem. 5.2) in the nonsingular $\left[\begin{array}{cc}S_{11} & S_{12} \\ S_{21} & O\end{array}\right]$ (see Lem. 5.1).

We arrive at the following algorithm for solving (4.24)-(4.26).

\section{Algorithm II.}

Step 1: Compute $\vec{f}_{\phi}$ of (5.5).

Step 2: Compute the right-hand side of (5.11).

Step 3: Solve (5.11) for $\vec{v}_{0, \text {. and }} \vec{v}_{1, \cdot}$

Step 4: Solve (5.6) for $\vec{u}$ and $\vec{v}$.

In the following subsections we explain how to solve linear systems with $S_{11}$ (involved in steps 2,4 ) and $S$ (involved in step 3).

\subsection{Solving systems with $S_{11}$}

Let vectors $\vec{u}, \vec{v}$, and $\vec{f}$ be of the forms (4.19), (4.21), and (4.27), respectively, and let

$$
\vec{g}=\left[g_{1,1}, \ldots, g_{1, N-1}, \ldots, g_{N-1,1}, \ldots, g_{N-1, N-1}\right]^{T} .
$$

Then, by (5.8), the system

$$
S_{11}[\vec{u}, \vec{v}]^{T}=[\vec{g}, \vec{f}]^{T}
$$

is equivalent to

$$
\begin{gathered}
\left(A_{\phi}^{\prime} \otimes B_{\psi, r}^{\prime}+B_{\phi}^{\prime} \otimes A_{\psi, r}^{\prime}\right)\left(M \otimes I_{N-3}\right) \vec{u}+\left(B_{\phi}^{\prime} \otimes B_{\psi, e}^{\prime}\right)\left(M \otimes I_{N+1}\right) \vec{v}=\vec{g} \\
\left(A_{\phi}^{\prime} \otimes B_{\psi, e}^{\prime}+B_{\phi}^{\prime} \otimes A_{\psi, e}^{\prime}\right)\left(M \otimes I_{N+1}\right) \vec{v}=\vec{f} .
\end{gathered}
$$


We rewrite $(5.15)$ as

$$
\begin{gathered}
\left(A_{\phi}^{\prime} \otimes B_{\psi}^{\prime}+B_{\phi}^{\prime} \otimes A_{\psi}^{\prime}\right)\left(M \otimes I_{N-1}\right) \vec{u}_{e}+\left(B_{\phi}^{\prime} \otimes B_{\psi, e}^{\prime}\right)\left(M \otimes I_{N+1}\right) \vec{v}=\vec{g} \\
\left(A_{\phi}^{\prime} \otimes B_{\psi, e}^{\prime}+B_{\phi}^{\prime} \otimes A_{\psi, e}^{\prime}\right)\left(M \otimes I_{N+1}\right) \vec{v}=\vec{f}, \\
B_{\psi}^{T} D B_{\psi} \vec{u}_{\cdot, 2}=\vec{\alpha}, \quad-B_{\psi}^{T} D B_{\psi} \vec{u} \cdot, 3=\vec{\beta},
\end{gathered}
$$

where

$$
\begin{gathered}
\vec{u}_{e}=\left[u_{2,2}, u_{2,3}, \ldots, u_{2, N}, \ldots, u_{N, 2}, u_{N, 3}, \ldots, u_{N, N}\right]^{T}, \\
\vec{u}_{\cdot, 2}=\left[u_{2,2}, u_{3,2} \ldots, u_{N, 2}\right]^{T}, \quad \vec{u}_{\cdot, 3}=\left[u_{2,3}, u_{3,3} \ldots, u_{N, 3}\right]^{T},
\end{gathered}
$$

and

$$
\vec{\alpha}=\left[\alpha_{2}, \ldots, \alpha_{N}\right]^{T}, \quad \vec{\beta}=\left[\beta_{2}, \ldots, \beta_{N}\right]^{T}
$$

Note that $\vec{u}_{e}$ in (5.17) is an extension of $\vec{u}$ in (4.19) with the components of $\vec{u}_{\cdot, 2}$ and $\vec{u}_{\cdot, 3}$ in (5.18) added to $\vec{u}$. Consequently, (5.16) is obtained from (5.15) by replacing $\vec{u}, A_{\psi, r}^{\prime}$, and $B_{\psi, r}^{\prime}$ with $\vec{u}_{e}, A_{\psi}^{\prime}$, and $B_{\psi}^{\prime}$, respectively, and by adding two additional equations for $\vec{u}_{\cdot, 2}$ and $\vec{u}_{\cdot, 3}$.

Note also that (5.14) (equivalently (5.15)) is a special case of (5.16) with $\vec{\alpha}=\vec{\beta}=\overrightarrow{0}$.

Lemma 5.4. The matrix of the linear system (5.16) is nonsingular.

Proof. The desired result follows easily from nonsingularity of $B_{\psi}^{T} D B_{\psi}$ and $S_{11}$.

Since $A_{\phi}^{\prime}$ and $B_{\phi}^{\prime}$ of (2.20) are symmetric and $A_{\phi}^{\prime}$ is positive definite, it follows from Corollary 8.7.2 in [9] that there exists a real nonsingular $(N-1) \times(N-1)$ matrix $Z$ and real

$$
\Lambda=\operatorname{diag}\left(\lambda_{2}, \ldots, \lambda_{N}\right)
$$

such that

$$
Z^{T} A_{\phi}^{\prime} Z=I_{N-1}, \quad Z^{T} B_{\phi}^{\prime} Z=\Lambda
$$

Since $Z$ of (5.21) and $M$ of (2.12) are nonsingular, (5.16) is equivalent to

$$
\begin{gathered}
\left(Z^{T} \otimes I_{N-1}\right)\left(A_{\phi}^{\prime} \otimes B_{\psi}^{\prime}+B_{\phi}^{\prime} \otimes A_{\psi}^{\prime}\right)\left(Z \otimes I_{N-1}\right) \vec{u}_{e}^{\prime}+\left(Z^{T} \otimes I_{N-1}\right)\left(B_{\phi}^{\prime} \otimes B_{\psi, e}^{\prime}\right)\left(Z \otimes I_{N+1}\right) \vec{v}^{\prime}=\vec{g}^{\prime} \\
\left(Z^{T} \otimes I_{N-1}\right)\left(A_{\phi}^{\prime} \otimes B_{\psi, e}^{\prime}+B_{\phi}^{\prime} \otimes A_{\psi, e}^{\prime}\right)\left(Z \otimes I_{N+1}\right) \vec{v}^{\prime}=\vec{f}^{\prime} \\
Z^{T} M^{-T} B_{\psi}^{T} D B_{\psi} M^{-1} Z \vec{u}_{\cdot, 2}^{\prime}=\vec{\alpha}^{\prime}, \quad-Z^{T} M^{-T} B_{\psi}^{T} D B_{\psi} M^{-1} Z \vec{u}_{\cdot, 3}^{\prime}=\vec{\beta}^{\prime}
\end{gathered}
$$

where $\vec{u}_{e}^{\prime}$ and $\vec{v}^{\prime}$ are such that

$$
\vec{u}_{e}=\left(W \otimes I_{N-1}\right) \vec{u}_{e}^{\prime}, \quad \vec{v}=\left(W \otimes I_{N+1}\right) \vec{v}^{\prime}, \quad \vec{u}_{\cdot, 2}=W \vec{u}_{\cdot, 2}^{\prime}, \quad \vec{u}_{\cdot, 3}=W \vec{u}_{\cdot, 3}^{\prime},
$$

with

$$
W=M^{-1} Z
$$


and

$$
\vec{g}^{\prime}=\left(Z^{T} \otimes I_{N-1}\right) \vec{g}, \quad \vec{f}^{\prime}=\left(Z^{T} \otimes I_{N-1}\right) \vec{f}, \quad \vec{\alpha}^{\prime}=W^{T} \vec{\alpha}, \quad \vec{\beta}^{\prime}=W^{T} \vec{\beta} .
$$

The vectors $\vec{u}_{e}^{\prime}, \vec{v}^{\prime}, \vec{u}_{\cdot, 2}^{\prime}, \vec{u}_{\cdot, 3}^{\prime}, \vec{g}^{\prime}, \vec{f}^{\prime}$, and $\vec{\alpha}^{\prime}, \vec{\beta}^{\prime}$ have the same forms as $\vec{u}_{e}$ of (5.17), $\vec{v}$ of (4.21), $\vec{u}_{., 2}, \vec{u}_{., 3}$ of (5.18), $\vec{g}$ of (5.13), $\vec{f}$ of (4.27), and $\vec{\alpha}, \vec{\beta}$ of (5.19), respectively. In the following, the components of the primed vectors are denoted by the primed letters corresponding to the unprimed vectors. For example,

$$
\vec{u}_{e}^{\prime}=\left[u_{2,2}^{\prime}, u_{2,3}^{\prime}, \ldots, u_{2, N}^{\prime}, \ldots, u_{N, 2}^{\prime}, u_{N, 3}^{\prime}, \ldots, u_{N, N}^{\prime}\right]^{T} .
$$

Using (2.19), (2.20), and (5.21) in (5.22), we obtain

$$
\begin{gathered}
\left(I_{N-1} \otimes B_{\psi}^{\prime}+\Lambda \otimes A_{\psi}^{\prime}\right) \vec{u}_{e}^{\prime}+\left(\Lambda \otimes B_{\psi, e}^{\prime}\right) \vec{v}^{\prime}=\vec{g}^{\prime} \\
\left(I_{N-1} \otimes B_{\psi, e}^{\prime}+\Lambda \otimes A_{\psi, e}^{\prime}\right) \vec{v}^{\prime}=\vec{f}^{\prime} \\
\Lambda \vec{u}_{\cdot, 2}^{\prime}=\vec{\alpha}^{\prime},-\Lambda \vec{u}_{\cdot, 3}^{\prime}=\vec{\beta}^{\prime}
\end{gathered}
$$

which, by (5.20), become

$$
\left(B_{\psi}^{\prime}+\lambda_{k} A_{\psi}^{\prime}\right) \vec{u}_{k, \cdot}^{\prime}+\lambda_{k} B_{\psi, e}^{\prime} \vec{v}_{k, \cdot}^{\prime}=\vec{g}_{k, \cdot}^{\prime}\left(B_{\psi, e}^{\prime}+\lambda_{k} A_{\psi, e}^{\prime}\right) \vec{v}_{k, \cdot}^{\prime}=\vec{f}_{k, \cdot}^{\prime}, u_{k, 2}^{\prime}=\alpha_{k}^{\prime} / \lambda_{k}, u_{k, 3}^{\prime}=-\beta_{k}^{\prime} / \lambda_{k},
$$

for $k=2, \ldots, N$, where

$$
\vec{u}_{k, \cdot}^{\prime}=\left[u_{k, 2}^{\prime}, \ldots, u_{k, N}^{\prime}\right]^{T}, \quad \vec{v}_{k, \cdot}^{\prime}=\left[v_{k, 0}^{\prime}, \ldots, v_{k, N}^{\prime}\right]^{T},
$$

and

$$
\vec{g}_{k, \cdot}^{\prime}=\left[g_{k, 1}^{\prime}, \ldots, g_{k, N-1}^{\prime}\right]^{T}, \quad \vec{f}_{k, \cdot}^{\prime}=\left[f_{k, 1}^{\prime}, \ldots, f_{k, N-1}^{\prime}\right]^{T} .
$$

Since $B_{\phi}^{\prime}$ is positive definite, it follows from the second equation in (5.21) that $\Lambda$ is also positive definite and hence, by (5.20), $\lambda_{k}>0, k=2, \ldots, N$. Clearly (5.26) is of the same form as (3.8) with $\lambda>0$.

It follows from (2.21) and the structure of $B_{\phi}^{\prime}$ (see Fig. 2) that the computation of $\Lambda$ and $Z$ satisfying (5.20) and (5.21) reduces to solving two symmetric eigenvalue problems with tridiagonal matrices. With the use of the $Q R$ algorithm for evaluating eigenvalues and the inverse iteration for evaluating the corresponding eigenvectors, $\Lambda$ and $Z$ can be precomputed with cost $O\left(N^{2}\right)$. Also $W$ of (5.24) can be precomputed with cost $O\left(N^{2}\right)$ since solving a linear system with $M$ requires $O(N)$ operations.

We are now in a position to formulate the following algorithm for solving (5.16).

\section{Algorithm III.}

Step 1: Compute $\vec{g}^{\prime}, \vec{f}^{\prime}, \vec{\alpha}^{\prime}$, and $\vec{\beta}^{\prime}$ using (5.25).

Step 2: For $k=2, \ldots, N$, solve (5.26) using Algorithm I of Section 3 for solving (3.8).

Step 3: Compute $\vec{u}_{e}$ and $\vec{v}$ using (5.23).

Steps 1 and 3 require $O\left(N^{3}\right)$ operations each while the cost of step 2 is $O\left(N^{2}\right)$. Hence the total cost of Algorithm III is $O\left(N^{3}\right)$.

In the remainder of this section we discuss the cost of Algorithm III for two special cases of (5.16).

In the first special case we assume that $\vec{\alpha}=\vec{\beta}=\overrightarrow{0}$ and that $\vec{g}, \vec{f}$ are such that

$$
g_{k, l}=f_{k, l}=0, \quad k=3, \ldots, N-1, \quad l=1, \ldots, N-1 .
$$

Also we assume that only components $\left\{u_{2, l}\right\}_{l=4}^{N}$ and $\left\{u_{3, l}\right\}_{l=4}^{N}$ of $\vec{u}_{e}$ need to be computed when solving (5.16). Let

$$
\vec{g}_{\cdot, l}=\left[g_{1, l}, \ldots, g_{N-1, l}\right]^{T}, \quad \vec{f}_{\cdot, l}=\left[f_{1, l}, \ldots, f_{N-1, l}\right]^{T}, \quad l=1, \ldots, N-1 .
$$


In this case, in step 1 of Algorithm III, for each $l=1, \ldots, N-1, Z^{T} \vec{g}_{\cdot, l}$ and $Z^{T} \vec{f}_{\cdot, l}$ are obtained by computing the corresponding liner combinations of the first 2 rows of $Z$. This can be done at a cost $O\left(N^{2}\right)$ for all $l$. As in the general case, the cost of step 2 remains $O\left(N^{2}\right)$. In step 3 , for each $l=4, \ldots, N, u_{2, l}$ and $u_{3, l}$ are obtained by computing the inner products of the first two rows of $W$ with $\left[u_{2, l}^{\prime}, \ldots, u_{N, l}^{\prime}\right]^{T}$. This can be done at a cost $O\left(N^{2}\right)$ for all $l$. Hence in this special case, the cost of Algorithm III is $O\left(N^{2}\right)$.

In the second special case we assume that $\vec{g}=\vec{f}=\overrightarrow{0}$ and that only components $\left\{v_{k, 0}\right\}_{k=4}^{N}$ and $\left\{v_{k, 1}\right\}_{l=4}^{N}$ of $\vec{v}$ need to be computed when solving (5.16). In step 1 of Algorithm III two multiplications by $W^{T}$ are performed to obtain $\vec{\alpha}^{\prime}$ and $\vec{\beta}^{\prime}$ at a cost $O\left(N^{2}\right)$. As in the general case, the cost of step 2 remains $O\left(N^{2}\right)$. In step 3 two multiplications by $W$ are performed to obtain $\left\{v_{k, 0}\right\}_{k=2}^{N}$ and $\left\{v_{k, 1}\right\}_{k=2}^{N}$ with cost $O\left(N^{2}\right)$. Hence, in this special case, the total cost of Algorithm III for computing the desired components of $\vec{v}$ is $O\left(N^{2}\right)$.

\subsection{Solving systems with $S$}

First, following the proof of Theorem 4.1 in [5], we show that the matrix $S$ of (5.12) is symmetric and positive definite. We start by proving the following lemma.

Lemma 5.5. Assume $U \in P_{N}^{0} \otimes P_{N}^{00}$ and $V \in P_{N} \otimes P_{N}$. Then

$$
\langle\langle\Delta U, V\rangle\rangle=\langle\langle U, \Delta V\rangle\rangle+\left.\sum_{j=1}^{N-1} w_{j}\left(U_{x} V\right)\left(\cdot, \xi_{j}\right)\right|_{-1} ^{1},
$$

where $\langle\langle\cdot, \cdot\rangle\rangle$ is defined in (4.4).

Proof. It follows from (2.4) and (2.2) that

$$
\sum_{i=1}^{N-1} w_{i}\left(p^{\prime \prime} q\right)\left(\xi_{i}\right)=\sum_{i=1}^{N-1} w_{i}\left(p q^{\prime \prime}\right)\left(\xi_{i}\right)+\left.\left(p^{\prime} q\right)\right|_{-1} ^{1}-\left.\left(p q^{\prime}\right)\right|_{-1} ^{1}, \quad p, q \in P_{N} .
$$

Hence, using (5.28) and $U(a, y)=0, a= \pm 1, y \in[-1,1]$, we have

$$
\sum_{i=1}^{N-1} w_{i}\left(U_{x x} V\right)\left(\xi_{i}, \xi_{j}\right)=\sum_{i=1}^{N-1} w_{i}\left(U V_{x x}\right)\left(\xi_{i}, \xi_{j}\right)+\left.\left(U_{x} V\right)\left(\cdot, \xi_{j}\right)\right|_{-1} ^{1}, \quad j=1, \ldots, N-1 .
$$

In a similar way, using (5.28) and $U(x, b)=U_{y}(x, b)=0, x \in[-1,1], b= \pm 1$, we obtain

$$
\sum_{j=1}^{N-1} w_{j}\left(U_{y y} V\right)\left(\xi_{i}, \xi_{j}\right)=\sum_{j=1}^{N-1} w_{j}\left(U V_{y y}\right)\left(\xi_{i}, \xi_{j}\right), \quad i=1, \ldots, N-1 .
$$

Multiplying (5.29) by $w_{j}, j=1, \ldots, N-1$, and summing with respect to $j$, and then multiplying (5.30) by $w_{i}$, $i=1, \ldots, N-1$, and summing with respect to $i$, we obtain the desired result.

Theorem 5.6. The matrix $S$ of (5.12) is symmetric and positive definite.

Proof. By (5.12), $S=S^{T}$ is equivalent to

$$
\left(S_{21} S_{11}^{-1} S_{12}\left[\vec{v}_{0, \cdot}^{(1)}, \vec{v}_{1, \cdot}^{(1)}\right]^{T},\left[\vec{v}_{0, \cdot}^{(2)}, \vec{v}_{1, \cdot}^{(2)}\right]^{T}\right)_{R^{2(N-3)}}=\left(S_{21} S_{11}^{-1} S_{12}\left[\vec{v}_{0, \cdot}^{(2)}, \vec{v}_{1, \cdot}^{(2)}\right]^{T},\left[\vec{v}_{0, \cdot}^{(1)}, \vec{v}_{1, \cdot}^{(1)}\right]^{T}\right)_{R^{2(N-3)}}
$$

for any

$$
\vec{v}_{0, \cdot}^{(n)}=\left[v_{0,4}^{(n)}, \ldots, v_{0, N}^{(n)}\right]^{T}, \quad \vec{v}_{1, \cdot}^{(n)}=\left[v_{1,4}^{(n)}, \ldots, v_{1, N}^{(n)}\right]^{T}, \quad n=1,2 .
$$


For $n=1,2$, let

$$
\vec{u}^{(n)}=\left[u_{2,4}^{(n)}, \ldots, u_{2, N}^{(n)}, \ldots, u_{N, 4}^{(n)}, \ldots, u_{N, N}^{(n)}\right]^{T}
$$

and

$$
\vec{v}^{(n)}=\left[v_{2,0}^{(n)}, \ldots, v_{2, N}^{(n)}, \ldots, v_{N, 0}^{(n)}, \ldots, v_{N, N}^{(n)}\right]^{T}
$$

be such that

$$
S_{11}\left[\vec{u}^{(n)}, \vec{v}^{(n)}\right]^{T}+S_{12}\left[\vec{v}_{0, \cdot}^{(n)}, \vec{v}_{2 N+1, \cdot}^{(n)}\right]^{T}=\overrightarrow{0}
$$

Then (5.31) becomes

$$
-\left(S_{21}\left[\vec{u}^{(1)}, \vec{v}^{(1)}\right]^{T},\left[\vec{v}_{0, \cdot}^{(2)}, \vec{v}_{1, \cdot}^{(2)}\right]^{T}\right)_{R^{2(N-3)}}=-\left(S_{21}\left[\vec{u}^{(2)}, \vec{v}^{(2)}\right]^{T},\left[\vec{v}_{0, \cdot}^{(1)}, \vec{v}_{1, \cdot}^{(1)}\right]^{T}\right)_{R^{2(N-3)}} .
$$

By (5.10), the last equation is the same as

$$
\begin{aligned}
& \left(D B_{\psi, r} \vec{u}_{3, \cdot}^{(1)}, B_{\psi, r} \vec{v}_{1, \cdot}^{(2)}\right)_{R^{N-1}}-\left(D B_{\psi, r} \vec{u}_{2, \cdot}^{(1)}, B_{\psi, r} \vec{v}_{0, \cdot}^{(2)}\right)_{R^{N-1}} \\
& \quad=\left(D B_{\psi, r} \vec{u}_{3, \cdot}^{(2)}, B_{\psi, r} \vec{v}_{1, \cdot}^{(1)}\right)_{R^{N-1}}-\left(D B_{\psi, r} \vec{u}_{2, \cdot}^{(2)}, B_{\psi, r} \vec{v}_{0, \cdot}^{(1)}\right)_{R^{N-1}}
\end{aligned}
$$

where, for $n=1,2$,

$$
\vec{u}_{2, \cdot}^{(n)}=\left[u_{2,4}^{(n)}, \ldots, u_{2, N}^{(n)}\right]^{T}, \quad \vec{u}_{3, \cdot}^{(n)}=\left[u_{3,4}^{(n)}, \ldots, u_{3, N}^{(n)}\right]^{T}
$$

To prove (5.33), we note, using (5.8) and (5.9), that (5.32) multiplied on the left by

$$
\left[\begin{array}{cc}
\left(B_{\phi}^{T} D\right)^{-1} \otimes\left(B_{\phi}^{T} D\right)^{-1} & O \\
O & \left(B_{\phi}^{T} D\right)^{-1} \otimes\left(B_{\phi}^{T} D\right)^{-1}
\end{array}\right]
$$

is the matrix-vector form of the spectral collocation problem

$$
-\Delta U^{(n)}\left(\xi_{i}, \xi_{j}\right)+V^{(n)}\left(\xi_{i}, \xi_{j}\right)=0, \quad-\Delta V^{(n)}\left(\xi_{i}, \xi_{j}\right)=0, \quad i, j=1, \ldots, N-1,
$$

where $U^{(n)}$ and $V^{(n)}$ are given by (4.15) and (4.17), respectively, with $u_{k, l}$ replaced by $u_{k, l}^{(n)}$ and $v_{k, l}$ replaced by $v_{k, l}^{(n)}$. Since $U^{(n)} \in P_{N}^{0} \otimes P_{N}^{00}$ and $V^{(n)} \in P_{N} \otimes P_{N}$, it follows from (5.34) and Lemma 5.5 that

$$
\left\langle\left\langle V^{(1)}, V^{(2)}\right\rangle\right\rangle=\left\langle\left\langle\Delta U^{(1)}, V^{(2)}\right\rangle\right\rangle=\left.\sum_{j=1}^{N-1} w_{j}\left(U_{x}^{(1)} V^{(2)}\right)\left(\cdot, \xi_{j}\right)\right|_{-1} ^{1} .
$$

In a similar way, we also have

$$
\left\langle\left\langle V^{(1)}, V^{(2)}\right\rangle\right\rangle=\left\langle\left\langle V^{(1)}, \Delta U^{(2)}\right\rangle\right\rangle=\left.\sum_{j=1}^{N-1} w_{j}\left(U_{x}^{(2)} V^{(1)}\right)\left(\cdot, \xi_{j}\right)\right|_{-1} ^{1},
$$

and hence

$$
\left.\sum_{j=1}^{N-1} w_{j}\left(U_{x}^{(1)} V^{(2)}\right)\left(\cdot, \xi_{j}\right)\right|_{-1} ^{1}=\left.\sum_{j=1}^{N-1} w_{j}\left(U_{x}^{(2)} V^{(1)}\right)\left(\cdot, \xi_{j}\right)\right|_{-1} ^{1}
$$


Using representations of $U^{(n)}$ and $V^{(n)}(c f .(4.15)$ and (4.17)), (2.8), and (2.14), it is easy to verify that (5.35) is the same as (5.33). This completes the proof of $S=S^{T}$.

To show that $S$ is positive definite, we observe, using the first part of the proof with $\vec{v}_{0, \cdot}^{(2)}=\vec{v}_{0, \cdot}^{(1)}$ and $\vec{v}_{1, .}^{(2)}=\vec{v}_{1, .}^{(1)}$, that

$$
\begin{aligned}
\left(S\left[\vec{v}_{0, \cdot}^{(1)}, \vec{v}_{1, \cdot}^{(1)}\right]^{T},\left[\vec{v}_{0, \cdot}^{(1)}, \vec{v}_{1, \cdot}^{(1)}\right]^{T}\right)_{R^{2(N-3)}} & =\left(D B_{\psi, r} \vec{u}_{3, \cdot}^{(1)}, B_{\psi, r} \vec{v}_{1, \cdot}^{(1)}\right)_{R^{N-1}}-\left(D B_{\psi, r} \vec{u}_{2, \cdot}^{(1)}, B_{\psi, r} \vec{v}_{0, \cdot}^{(1)}\right)_{R^{N-1}} \\
& =\left.\sum_{j=1}^{N-1} w_{j}\left(U_{x}^{(1)} V^{(1)}\right)\left(\cdot, \xi_{j}\right)\right|_{-1} ^{1}=\left\langle\left\langle V^{(1)}, V^{(1)}\right\rangle\right\rangle,
\end{aligned}
$$

which shows that $S$ is nonnegative definite. Since $S=S^{T}$ and $S$ is nonsingular (see Lem. 5.3), it follows that $S$ is positive definite.

It follows from Theorem 5.6 that the PCG method is a good candidate for solving the linear system with $S$. Therefore, in the following, we discuss matrix-vector multiplications involving $S$, the selection of a preconditioner, and the solution of a linear system with this preconditioner.

It follows from (5.12) that in order to multiply by $S$, we have to first multiply by $S_{12}$, then solve with $S_{11}$, and finally multiply by $S_{21}$. Let $\vec{v}_{0,}$. and $\vec{v}_{1}$. be of the forms given in (4.22) and let

$$
[\vec{g}, \vec{f}]^{T}=S_{12}\left[\vec{v}_{0, \cdot}, \vec{v}_{1,}\right]^{T},
$$

where $\vec{g}$ and $\vec{f}$ have the forms given in (5.13) and (4.27), respectively. Then, by (5.9),

$$
\vec{g}=\left(B_{\psi, t}^{\prime} \otimes I_{N-1}\right)\left(I_{2} \otimes B_{\psi, r}^{\prime}\right)\left[\vec{v}_{0, .}, \vec{v}_{1, \cdot}\right]^{T}
$$

and

$$
\vec{f}=\left(B_{\psi, t}^{\prime} \otimes I_{N-1}\right)\left(I_{2} \otimes A_{\psi, r}^{\prime}\right)\left[\vec{v}_{0, \cdot}, \vec{v}_{1,},\right]^{T} .
$$

Hence the computation of $\vec{g}$ and $\vec{f}$ requires 2 multiplications by $B_{\psi, r}^{\prime}, 2$ multiplications by $A_{\psi, r}^{\prime}$, and $2(N-1)$ multiplications by $B_{\psi, t}^{\prime}$. It follows from $(2.22),(2.15),(4.23),(5.4)$ that $A_{\psi}^{\prime}=\left[\because \because \mid A_{\psi, r}^{\prime}\right]$ and $B_{\psi}^{\prime}=\left[\because: \mid B_{\psi, r}^{\prime}\right]$, where the symbol $\because:$ denotes the first two columns of the matrix appearing on the left-hand side. Hence the products of $A_{\psi, r}^{\prime}$ and $B_{\psi, r}^{\prime}$ with a vector can be obtained by computing the products of $A_{\psi}^{\prime}$ and $B_{\psi}^{\prime}$ with the augmented vector whose first two components are set to zero. By (2.23), (2.21), and the structures of $M$ and $B_{\phi}^{\prime}$ (see Figs. 1 and 2), all the required multiplications by $A_{\psi, r}^{\prime}$ and $B_{\psi, r}^{\prime}$ involve $O(N)$ operations. It also follows from the structure of $B_{\psi, t}^{\prime}$ (see Fig. 3) that all the required multiplications by $B_{\psi, t}^{\prime}$ take $O(N)$ operations. Hence the total cost of multiplying by $S_{12}$ is $O(N)$.

With $\vec{u}$ and $\vec{v}$ of the forms (4.19) and (4.21), it remains to solve (5.14) and then compute $S_{21}[\vec{u}, \vec{v}]^{T}$. Note that only the subvectors $\vec{u}_{2,}$. and $\vec{u}_{3,}$. of $\vec{u}$ are needed for multiplication by $S_{21}$ of (5.10). Moreover, (5.36), (5.37), and the structure of $B_{\psi, t}^{\prime}$ (see Fig. 3) imply that the components of $\vec{g}$ and $\vec{f}$ satisfy (5.27). Hence, it follows from the discussion in Section 5.2 of the first special case of (5.16) that computing $\vec{u}_{2, \text {. and }} \vec{u}_{3, \text {, requires }}$

$O\left(N^{2}\right)$ operations. Finally, (2.15) and (4.23) imply that $B_{\psi}^{T} D B_{\psi}=\left[\because: \mid B_{\psi, r}\right]^{T} D\left[\because: \mid B_{\psi, r}\right]$. But (2.19) and (2.20)

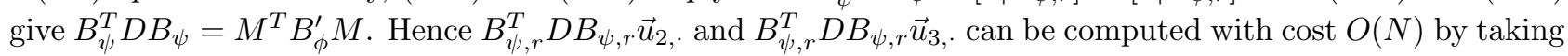
advantage of the structures of $M$ and $B_{\phi}^{\prime}$ (see Figs. 1 and 2).

Thus the total cost of multiplying a vector by $S$ is $O\left(N^{2}\right)$.

In the remainder of this section we select a preconditioner for $S$ and discuss the solution of a linear system with this preconditioner. First, interchanging the roles of the $x$ and $y$ coordinates and replacing $\vec{u}_{e}, \vec{u}_{, 2}, \vec{u}_{, 3}$, 
and $\vec{v}$ with $\vec{w}, \vec{w}_{2, .}, \vec{w}_{3, .}$, and $\vec{z}$, respectively, we rewrite (5.16) with $\vec{g}=\vec{f}=\overrightarrow{0}$ to obtain

$$
\begin{gathered}
\left(A_{\psi}^{\prime} \otimes B_{\phi}^{\prime}+B_{\psi}^{\prime} \otimes A_{\phi}^{\prime}\right)\left(I_{N-1} \otimes M\right) \vec{w}+\left(B_{\psi, e}^{\prime} \otimes B_{\phi}^{\prime}\right)\left(I_{N+1} \otimes M\right) \vec{z}=\overrightarrow{0}, \\
\left(A_{\psi, e}^{\prime} \otimes B_{\phi}^{\prime}+B_{\psi, e}^{\prime} \otimes A_{\phi}^{\prime}\right)\left(I_{N+1} \otimes M\right) \vec{z}=\overrightarrow{0}, \\
B_{\psi}^{T} D B_{\psi} \vec{w}_{2, .}=\vec{\alpha}, \quad-B_{\psi}^{T} D B_{\psi} \vec{w}_{3, .}=\vec{\beta},
\end{gathered}
$$

where

$$
\begin{gathered}
\vec{w}=\left[w_{2,2}, w_{2,3}, \ldots, w_{2, N}, \ldots, w_{N, 2}, w_{N, 3}, \ldots, w_{N, N}\right]^{T}, \\
\vec{w}_{2, \cdot}=\left[w_{2,2}, w_{2,3} \ldots, w_{2, N}\right]^{T}, \quad \vec{w}_{3, \cdot}=\left[w_{3,2}, w_{3,3} \ldots, w_{3, N}\right]^{T}, \\
\vec{z}=\left[z_{0,2}, \ldots, z_{0, N}, \ldots, z_{N, 2}, \ldots, z_{N, N}\right]^{T} .
\end{gathered}
$$

(Of course solving (5.38) is equivalent to solving (5.16) with $\vec{g}=\vec{f}=\overrightarrow{0}$.) We split the vector $\vec{z}$ of (5.39) into two parts,

$$
\vec{z}_{r}=\left[z_{2,2}, \ldots, z_{2, N}, \ldots, z_{N, 2}, \ldots, z_{N, N}\right]^{T}
$$

and

$$
\vec{z}_{0, \cdot}=\left[z_{0,2}, \ldots, z_{0, N}\right]^{T}, \quad \vec{z}_{1, \cdot}=\left[z_{1,2}, \ldots, z_{1, N}\right]^{T} .
$$

(The vector $\vec{z}_{r}$ can be viewed as a restriction of $\vec{z}$ with the components of $\vec{z}_{0,}$. and $\vec{z}_{1}$, being removed from $\vec{z}$.) Then (5.38) can be written as

$$
\begin{gathered}
P_{11}\left[\vec{w}, \vec{z}_{r}\right]^{T}+P_{12}\left[\vec{z}_{0, \cdot}, \vec{z}_{1,} \cdot\right]^{T}=\overrightarrow{0}, \\
P_{21}\left[\vec{w}, \vec{z}_{r}\right]^{T}=[\vec{\alpha}, \vec{\beta}]^{T},
\end{gathered}
$$

where

$$
P_{11}=\left[\begin{array}{cc}
\left(A_{\psi}^{\prime} \otimes B_{\phi}^{\prime}+B_{\psi}^{\prime} \otimes A_{\phi}^{\prime}\right)\left(I_{N-1} \otimes M\right) & \left(B_{\psi}^{\prime} \otimes B_{\phi}^{\prime}\right)\left(I_{N-1} \otimes M\right) \\
O & \left(A_{\psi}^{\prime} \otimes B_{\phi}^{\prime}+B_{\psi}^{\prime} \otimes A_{\phi}^{\prime}\right)\left(I_{N-1} \otimes M\right)
\end{array}\right]
$$

$P_{12}$ is the block multiplying $\left[\vec{z}_{0, .}, \vec{z}_{1, .}\right]^{T}$, and

$$
P_{21}=\left[\begin{array}{ccc}
B_{\psi}^{T} D B_{\psi} & O & O \\
O & -B_{\psi}^{T} D B_{\psi} & O
\end{array}\right] .
$$

Note that the two blocks $B_{\psi}^{T} D B_{\psi}$ in $P_{21}$ correspond to multiplications by $\vec{w}_{2, \text {. }}$ and $\vec{w}_{3, .}$

Lemma 5.7. The matrix $P_{11}$ of (5.41) is nonsingular.

Proof. With $\vec{u}_{e}$ of the form (5.17), the equation $\left(A_{\psi} \otimes B_{\psi}+B_{\psi} \otimes B_{\psi}\right) \vec{u}_{e}=\overrightarrow{0}$ is the matrix-vector representation of the following spectral collocation problem: find $U \in P_{N}^{0} \otimes P_{N}^{0}$ such that

$$
-\Delta U\left(\xi_{i}, \xi_{j}\right)=0, \quad i, j=1, \ldots, N-1 .
$$

It can be shown, using an approach similar to the proof of Theorem 4.3, that the only solution to this problem is $U=0$ which implies the nonsingularity of $\left(A_{\psi} \otimes B_{\psi}+B_{\psi} \otimes A_{\psi}\right)$. Hence this, (2.22), (2.20), (2.19), and the nonsingularity of $B_{\phi}^{T} D$ imply the nonsingularity of $P_{11}$. 
Since $P_{11}$ is nonsingular, eliminating $\left[\vec{w}, \vec{z}_{r}\right]^{T}$ from $(5.40)$, we obtain

$$
P\left[\vec{z}_{0, \cdot}, \vec{z}_{1, \cdot}\right]^{T}=-[\vec{\alpha}, \vec{\beta}]^{T}
$$

where the $2(N-1) \times 2(N-1)$ Schur complement matrix

$$
P=P_{21} P_{11}^{-1} P_{12}
$$

Theorem 5.8. The matrix $P$ is symmetric and positive definite.

Proof. The proof of this theorem is similar to that of Theorem 5.6. First we observe that $P$ is nonsingular since it is the Schur complement of the nonsingular $P_{11}$ (see Lem. 5.7) in the nonsingular $\left[\begin{array}{cc}P_{11} & P_{12} \\ P_{21} & O\end{array}\right]$ (see Lem. 5.4). Then we prove that $P=P^{T}$ and that $P$ is nonnegative definite. This and the nonsingularity of $P$ imply that $P$ is positive definite.

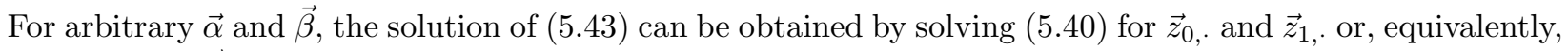
(5.16) with $\vec{g}=\vec{f}=\overrightarrow{0}$, for the components $\left\{v_{k, 0}\right\}_{k=2}^{N}$ and $\left\{v_{k, 1}\right\}_{k=2}^{N}$ of $\vec{v}$.

As a preconditioner for $S$ we take the $2(N-3) \times 2(N-3)$ matrix $\tilde{P}$ which arises from eliminating $z_{0,2}, z_{0,3}$, $z_{1,2}$, and $z_{1,3}$ in (5.43). Clearly such a $\tilde{P}$ is symmetric and positive definite, being the Schur complement of a symmetric and positive definite submatrix in the symmetric and positive definite $P$. Moreover, for arbitrary $\left\{\alpha_{k}\right\}_{k=4}^{N}$ and $\left\{\beta_{k}\right\}_{k=4}^{N}$, the solution of the system

$$
\tilde{P}\left[z_{0,4}, \ldots, z_{0, N}, z_{1,4}, \ldots, z_{1, N}\right]^{T}=-\left[\alpha_{4}, \ldots, \alpha_{N}, \beta_{4}, \ldots, \beta_{N}\right]^{T}
$$

can be obtained by solving (5.43), with $\alpha_{2}=\alpha_{3}=\beta_{2}=\beta_{3}=0$, for $\left\{z_{0, k}\right\}_{k=4}^{N}$ and $\left\{z_{1, k}\right\}_{k=4}^{N}$. Hence, we find the solution of (5.45) by solving (5.16) with $\vec{g}=\vec{f}=\overrightarrow{0}$ and $\alpha_{2}=\alpha_{3}=\beta_{2}=\beta_{3}=0$, for the components $\left\{v_{k, 0}\right\}_{k=4}^{N}$ and $\left\{v_{k, 1}\right\}_{k=4}^{N}$ of $\vec{v}$. It follows from the discussion in Section 5.2 of the second special case of (5.16) that the cost of computing these components is $O\left(N^{2}\right)$.

Finally, we explain how to select the functions $\psi_{2}$ and $\psi_{3}$ of (2.9) and (2.8). This selection is motivated by making

$$
\kappa_{2}\left(\tilde{P}^{-1 / 2} S \tilde{P}^{-1 / 2}\right)=\lambda_{\max }\left(\tilde{P}^{-1} S\right) / \lambda_{\min }\left(\tilde{P}^{-1} S\right)
$$

independent of $N$, where for symmetric and positive definite $A, \kappa_{2}(A)=\lambda_{\max }(A) / \lambda_{\min }(A)$. Equations (5.12) and (5.10) imply that

$$
S=\left[\begin{array}{cc}
\Psi_{r} & O \\
O & \Psi_{r}
\end{array}\right]\left[\begin{array}{ll}
R^{(1)} & R^{(2)} \\
R^{(3)} & R^{(4)}
\end{array}\right]
$$

where

$$
\Psi_{r}=B_{\psi, r}^{T} D B_{\psi, r}, \quad\left[\begin{array}{ll}
R^{(1)} & R^{(2)} \\
R^{(3)} & R^{(4)}
\end{array}\right]=\left[\begin{array}{ccc}
I_{N-3} & O & O \\
O & -I_{N-3} & O
\end{array}\right] S_{11}^{-1} S_{12}
$$

In a similar way, using (5.44) and (5.42), we have

$$
P=\left[\begin{array}{ll}
\Psi & O \\
O & \Psi
\end{array}\right]\left[\begin{array}{ll}
Q^{(1)} & Q^{(2)} \\
Q^{(3)} & Q^{(4)}
\end{array}\right]
$$


TABLE 1. $\kappa_{2}\left(\tilde{P}^{-1 / 1} S . \tilde{P}^{-1 / 2}\right)$

\begin{tabular}{|cc|}
\hline$N$ & $\kappa_{2}\left(\tilde{P}^{-1 / 2} S \tilde{P}^{-1 / 2}\right)$ \\
\hline 10 & 1.56 \\
20 & 1.63 \\
30 & 1.65 \\
40 & 1.66 \\
50 & 1.67 \\
60 & 1.67 \\
70 & 1.67 \\
\hline
\end{tabular}

where

$$
\Psi=B_{\psi}^{T} D B_{\psi}, \quad\left[\begin{array}{ll}
Q^{(1)} & Q^{(2)} \\
Q^{(3)} & Q^{(4)}
\end{array}\right]=\left[\begin{array}{ccc}
I_{N-1} & O & O \\
O & -I_{N-1} & O
\end{array}\right] P_{11}^{-1} P_{12}
$$

It follows from (2.15), (4.23), (2.1), and (2.2) that

$$
\Psi=\left[\begin{array}{ll}
\Psi_{11} & \Psi_{12} \\
\Psi_{12}^{T} & \Psi_{r}
\end{array}\right]
$$

where

$$
\Psi_{11}=\left[\begin{array}{cc}
\left\langle\psi_{2}, \psi_{2}\right\rangle & \left\langle\psi_{2}, \psi_{3}\right\rangle \\
\left\langle\psi_{3}, \psi_{2}\right\rangle & \left\langle\psi_{3}, \psi_{3}\right\rangle
\end{array}\right], \quad \Psi_{12}=\left[\begin{array}{ccc}
\left\langle\psi_{2}, \psi_{4}\right\rangle & \cdots & \left\langle\psi_{2}, \psi_{N-1}\right\rangle \\
\left\langle\psi_{3}, \psi_{4}\right\rangle & \cdots & \left\langle\psi_{3}, \psi_{N-1}\right\rangle
\end{array}\right]
$$

If $\Psi_{12}=0$, then substituting (5.47) into (5.43) and eliminating $\left[z_{0,2}, z_{0,3}\right]^{T},\left[z_{1,2}, z_{1,3}\right]^{T}$, we see that $\tilde{P}$ has the form

$$
\tilde{P}=\left[\begin{array}{cc}
\Psi_{r} & O \\
O & \Psi_{r}
\end{array}\right]\left[\begin{array}{ll}
\tilde{Q}^{(1)} & \tilde{Q}^{(2)} \\
\tilde{Q}^{(3)} & \tilde{Q}^{(4)}
\end{array}\right]
$$

Therefore, by (5.46) and (5.48), the block $\left[\begin{array}{cc}\Psi_{r} & O \\ O & \Psi_{r}\end{array}\right]$ is canceled in $\tilde{P}^{-1} S$. For $\psi_{2}$ and $\psi_{3}$ of (2.9) satisfying (2.8) and

$$
\left\langle\psi_{2}, \psi_{k}\right\rangle=0, \quad\left\langle\psi_{3}, \psi_{k}\right\rangle=0, \quad k=4, \ldots, N,
$$

the results of Table 1 show that $\kappa_{2}\left(\tilde{P}^{-1 / 2} S \tilde{P}^{-1 / 2}\right)$ is bounded from above by a positive constant which is independent of $N$. For the simpler choice

$$
\psi_{2}(x)=\frac{1}{10}\left[L_{3}(x)-L_{1}(x)\right]-\frac{1}{6}\left[L_{2}(x)-L_{0}(x)\right], \quad \psi_{3}(x)=\frac{1}{10}\left[L_{3}(x)-L_{1}(x)\right]+\frac{1}{6}\left[L_{2}(x)-L_{0}(x)\right],
$$

$\kappa_{2}\left(\tilde{P}^{-1 / 2} S \tilde{P}^{-1 / 2}\right)$ grows rapidly as $N \rightarrow \infty$ since the block $\left[\begin{array}{cc}\Psi_{r} & O \\ O & \Psi_{r}\end{array}\right]$ is not canceled in $\tilde{P}^{-1} S$ and $\kappa_{2}\left(\Psi_{r}\right)$ grows rapidly as $N \rightarrow \infty$. 


$$
\left[\begin{array}{ccccccc}
\times & \times & \times & & & & \\
\times & \times & \times & \times & & & \\
& \times & \times & \times & \times & & \\
& & \ddots & \ddots & \ddots & \ddots & \\
& & & \times & \times & \times & \times \\
& & & & \times & \times & \times \\
\times & \times & \cdots & \times & \times & \times & \times
\end{array}\right]
$$

Figure 5. Structure of the matrices in decoupled systems for (5.50).

It follows from $(2.9),(5.49),(2.8),(2.11),\left\langle\phi_{k}, \phi_{l}\right\rangle=0, k, l=2, \ldots, N, l \neq k, k \pm 2$, and $\phi_{k}^{\prime}(-1)=$ $(-1)^{k-1} \phi_{k}^{\prime}(1), k=2, \ldots, N$, that finding $\left\{\alpha_{k}\right\}_{k=2}^{N}$ is equivalent to solving the system

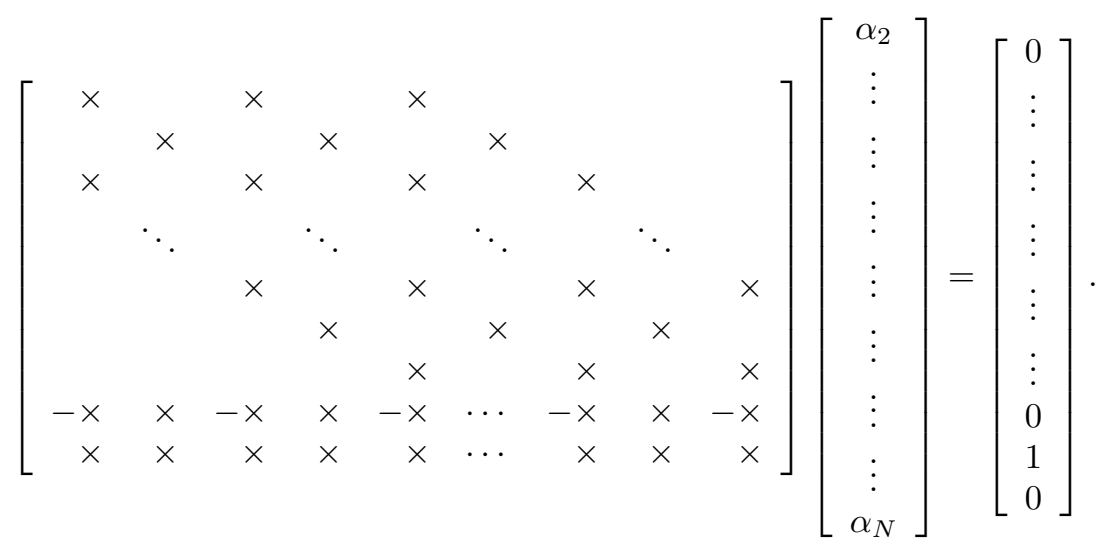

For $\left\{\beta_{k}\right\}_{k=2}^{N}$ the last two components on the right-hand side are to be switched which implies (2.10). System (5.50) can be decoupled into two systems, one for $\alpha_{k}$ with even $k$ and the other for $\alpha_{k}$ with odd $k$. The matrices in these two systems have the structure shown in Figure 5 and hence each system can be solved with cost $O(N)$.

\subsection{Cost of solving the biharmonic spectral collocation problem}

We now give the cost of solving (4.24)-(4.26) using Algorithm II of Section 5.1.

As discussed in Section 5.2, we precompute $\Lambda$ and $Z$ of (5.20) and (5.21) and $W$ of (5.24) with cost $O\left(N^{2}\right)$. Also, as discussed in Section 5.3, we precompute $\left\{\alpha_{k}\right\}_{k=2}^{N}$ in $(2.9)$ with cost $O(N)$.

Step 1 of Algorithm II involves computing $\vec{f}_{\phi}$ of (5.5) and it requires $O\left(N^{3}\right)$ operations since $B_{\phi}$ is full.

Step 2 involves solving (5.14) with $\vec{g}=\overrightarrow{0}$ and then computing $S_{21}[\vec{u}, \vec{v}]^{T}$. Only the subvectors $\vec{u}_{2, \text { and }} \vec{u}_{3 \text {, of }}$ $\vec{u}$ are needed when solving (5.14). These subvectors are computed with cost $O\left(N^{3}\right)$ since the cost of computing $\overrightarrow{f^{\prime}}$ in step 1 of Algorithm III is $O\left(N^{3}\right)$. (Note that $\vec{g}^{\prime}, \vec{\alpha}^{\prime}, \vec{\beta}^{\prime}$ need not be computed in step 1 of Algorithm III since they are $\overrightarrow{0}$. Also, in step 3 of Algorithm III, $\vec{u}_{2}$. and $\vec{u}_{3}$. can be computed with cost $O\left(N^{2}\right)$.) Then, it follows from the discussion in Section 5.3 that the cost of computing $S_{21}[\vec{u}, \vec{v}]^{T}$ is $O(N)$. Thus the cost of step 2 is $O\left(N^{3}\right)$.

Step 3 is carried out using the PCG method with $\tilde{P}$ as a preconditioner for $S$. It follows from Section 5.3 that the cost of each PCG iteration, involving multiplication by $S$ and solution with $\tilde{P}$, is $O\left(N^{2}\right)$. Hence with the number of the PCG iterations proportional to $\log N$ the cost of step 3 is $O\left(N^{2} \log N\right)$. 
TABLE 2. Maximum absolute error for numerical example.

\begin{tabular}{|ccc|}
\hline$N$ & $\|u-U\|_{\infty}$ & $\|v-V\|_{\infty}$ \\
\hline 12 & $0.11(+1)$ & $0.11(+3)$ \\
16 & $0.25(-1)$ & $0.44(+1)$ \\
20 & $0.26(-3)$ & $0.59(-1)$ \\
24 & $0.16(-5)$ & $0.36(-3)$ \\
28 & $0.45(-8)$ & $0.11(-5)$ \\
32 & $0.71(-11)$ & $0.16(-8)$ \\
\hline
\end{tabular}

TABLE 3. CPU times.

\begin{tabular}{|cc|}
\hline$N$ & CPU time (secs) \\
\hline 16 & 0.52 \\
32 & 3.09 \\
48 & 9.59 \\
64 & 21.23 \\
\hline
\end{tabular}

In step 4 , we first compute $S_{12}\left[\vec{v}_{0, .}, \vec{v}_{1, \cdot}\right]^{T}$ and then solve with $S_{11}$. It follows from Section 5.3 that the cost of computing $S_{12}\left[\vec{v}_{0, \cdot}, \vec{v}_{1, \cdot}\right]^{T}$ is $O(N)$. The cost of solving with $S_{11}$ is $O\left(N^{3}\right)$ since the cost of computing $\vec{f}^{\prime}$ in step 1 of Algorithm III is $O\left(N^{3}\right)$. (Note that in step 1 of Algorithm III, $\vec{g}^{\prime}$ can be computed with cost $O\left(N^{2}\right)$ since it follows from (5.36) and the structure of $B_{\psi, t}^{\prime}$ (see Fig. 3) that the components of $\vec{g}$ are as in (5.27). Of course $\vec{\alpha}^{\prime}=\vec{\beta}^{\prime}=\overrightarrow{0}$ and hence they need not be computed.) Hence step 4 costs $O\left(N^{3}\right)$.

Therefore, the total cost of solving the spectral collocation problem is $O\left(N^{3}\right)$.

\section{Numerical Results}

We solved (1.1) with

$$
f(x, y)=128 \pi^{4}\left[\cos (4 \pi x) \cos (4 \pi y)-\sin ^{2}(2 \pi x) \cos (4 \pi y)-\cos (4 \pi x) \sin ^{2}(2 \pi y)\right] .
$$

The exact solution of this problem, which was also considered by Shen [17] and Bjørstad and Tjøstheim [6], is $u=\sin ^{2}(2 \pi x) \sin ^{2}(2 \pi y)$. The number of iterations in the PCG part of our method was taken to be $2 \log N$. In Table 2 we present the maximum absolute error in $u$ and $v=\Delta u$ on a uniform $(0.02) \times(0.02)$ grid for different values of $N$. The exponential convergence achieved is shown in Figure 6 where we present the graph of the logarithm of the maximum absolute error versus N. In Table 3, we present the CPU times required for the solution of the problem on a RS6000-250 workstation for various values of $N$, including the cost of precomputing the matrices $\Lambda, Z, W$, and the coefficients $\left\{\alpha_{k}\right\}_{k=2}^{N}$. From the table it is clear that the CPU times grow roughly like $N^{3}$.

\section{Conclusions}

In this study we considered the numerical solution of the biharmonic Dirichlet problem on a square by a Legendre spectral collocation method. A mixed formulation approach was used to rewrite the biharmonic equation as a system of two coupled Poisson's equations for the unknown solution and its Laplacian. The solution of the Legendre spectral collocation problem for the two Poisson's equations was reduced to the solution of a 


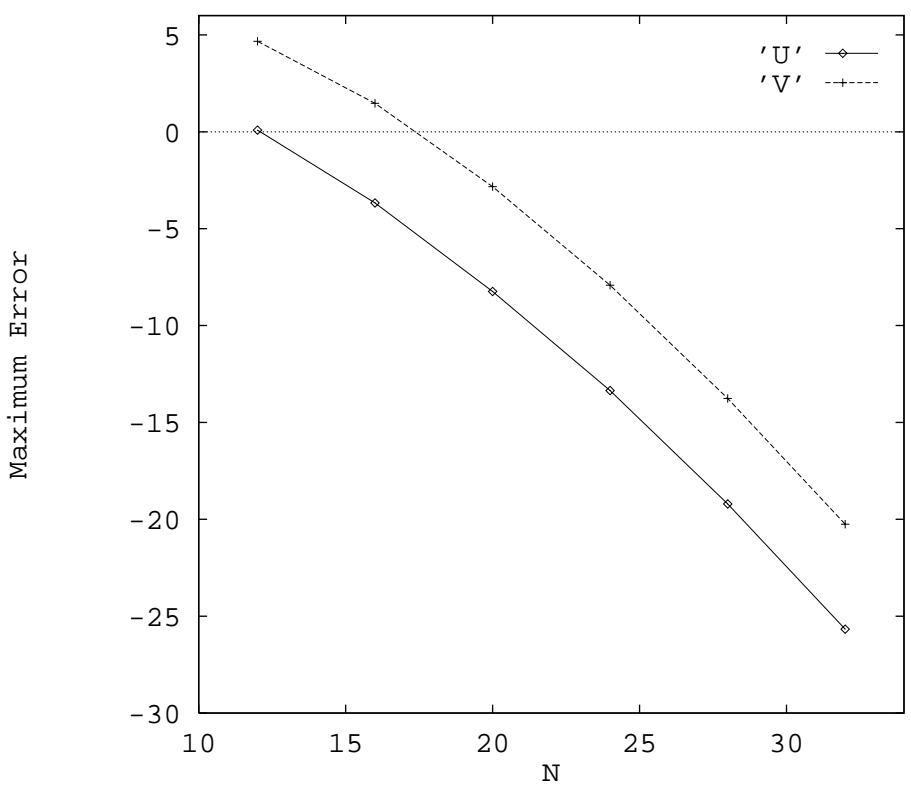

FIGURE 6. Logarithm of the maximum absolute error versus $\mathrm{N}$.

Schur complement system for the Laplacian of the approximate solution on two vertical sides of the square. Since the Schur complement matrix is symmetric and positive definite, the Schur system was solved by the PCG method with the preconditioner obtained from an auxiliary problem. The total cost of the proposed algorithm is $O\left(N^{3}\right)$ which is comparable to the cost of state-of-the-art spectral Galerkin methods. An important advantage of the mixed formulation approach is that in addition to an approximation of the solution, we also obtain automatically an approximation to its Laplacian. The spectral convergence of these two approximations is demonstrated numerically on a test problem from the literature.

The extension of the proposed method to complex geometries is presently under investigation. So far we have examined the application of the domain decomposition approach to the formulation and solution of a Legendre spectral collocation problem for Poisson's equation on a $L$-shaped region decomposed into three rectangles. The approximate solution is continuous throughout the region and its normal derivatives coincide at the collocation points on two interfaces. Firstly, the self-adjoint and positive definite approximate problem on the interfaces is solved using the PCG method. Subsequently, three independent approximate problems on three rectangles are solved efficiently using a matrix decomposition technique similar to the one described in this paper. We hope that a similar domain decomposition approach will allow us to formulate and solve a Legendre spectral collocation problem for the biharmonic equation on a $L$-shaped region and, in general, on regions which are unions of rectangles with sides parallel to the coordinate axes.

Acknowledgements. The work of the first author was supported in part by National Science Foundation grant DMS9805827. Parts of this work were performed while the second author was a Visiting Associate Professor in the Department of Mathematical and Computer Sciences, Colorado School of Mines, Golden, Colorado 80401, U.S.A. 


\section{REFERENCES}

[1] C. Bernardi and Y. Maday, Spectral methods for the approximation of fourth order problems: Applications to the Stokes and Navier-Stokes equations. Comput. and Structures 30 (1988) 205-216.

[2] C. Bernardi and Y. Maday, Some spectral approximations of one-dimensional fourth order problems, in: Progress in Approximation Theory, P. Nevai and A. Pinkus Eds., Academic Press, San Diego (1991), 43-116.

[3] C. Bernardi and Y. Maday, Spectral methods, in Handbook of Numerical Analysis, Vol. V, Part 2: Techniques of Scientific Computing, P.G. Ciarlet and J.L. Lions Eds., North-Holland, Amsterdam (1997) 209-485.

[4] C. Bernardi, G. Coppoletta and Y. Maday, Some spectral approximations of two-dimensional fourth order problems. Math. Comp. 59 (1992) 63-76.

[5] B. Bialecki, A fast solver for the orthogonal spline collocation solution of the biharmonic Dirichlet problem on rectangles. submitted.

[6] P.E. Bjørstad and B.P. Tjøstheim, Efficient algorithms for solving a fourth-order equation with the spectral-Galerkin method. SIAM J. Sci. Comput. 18 (1997) 621-632.

[7] J.P. Boyd, Chebyshev and Fourier Spectral Methods. Springer-Verlag, Berlin (1989).

[8] J. Douglas Jr. and T. Dupont, Collocation Methods for Parabolic Equations in a Single Space Variable. Lect. Notes Math. 358, Springer-Verlag, New York, 1974.

[9] G.H. Golub and C.F. van Loan, Matrix Computations, Third edn., The Johns Hopkins University Press, Baltimore, MD (1996).

[10] W. Heinrichs, A stabilized treatment of the biharmonic operator with spectral methods. SIAM J. Sci. Stat. Comput. 12 (1991) $1162-1172$.

[11] A. Karageorghis, The numerical solution of laminar flow in a re-entrant tube geometry by a Chebyshev spectral element collocation method. Comput. Methods Appl. Mech. Engng. 100 (1992) 339-358.

[12] A. Karageorghis, A fully conforming spectral collocation scheme for second and fourth order problems. Comput. Methods Appl. Mech. Engng. 126 (1995) 305-314.

[13] A. Karageorghis and T.N. Phillips, Conforming Chebyshev spectral collocation methods for the solution of laminar flow in a constricted channel. IMA Journal Numer. Anal. 11 (1991) 33-55.

[14] A. Karageorghis and T. Tang, A spectral domain decomposition approach for steady Navier-Stokes problems in circular geometries. Computers and Fluids 25 (1996) 541-549.

[15] Z.-M. Lou, B. Bialecki, and G. Fairweather, Orthogonal spline collocation methods for biharmonic problems. Numer. Math. 80 (1998) 267-303.

[16] W.W. Schultz, N.Y. Lee and J.P. Boyd, Chebyshev pseudospectral method of viscous flows with corner singularities. J. Sci. Comput. 4 (1989) 1-19.

[17] J. Shen, Efficient spectral-Galerkin method I. Direct solvers of second- and forth-order equations using Legendre polynomials. SIAM J. Sci. Comput. 15 (1994) 1489-1505. 